\title{
Computational Study of No-slip and Rarefied Slip Flows in Infinite Structured Porous Media
}

\author{
Sridhar Palle*, and Shahrouz Aliabadi \\ Northrop Grumman Center for High Performance Computing \\ Jackson State University, Jackson, MS, USA
}

\begin{abstract}
No-slip and rarefied slip flows in infinite structured porous media are numerically investigated in this study using hybrid FE/FV methods. The hybrid FE/FV flow solver methodology is based on the pressure correction method which involves a fractional step approach to first obtain an intermediate velocity by solving the momentum equations with the matrix-free, implicit, cell-centered finite volume method. The poisson equation resulting from the fractional step approach is then solved by node based Galerkin finite element method for an auxiliary variable which is closely related to pressure and is used to update the velocity and pressure fields.
\end{abstract}

Three dimensional numerical simulations are conducted using our hybrid FE/FV flow solver for analyzing fluid flow in infinite structured porous media. In this study, structured porous media is represented by packed beds of spherical particles in different packing configurations including Simple (SC), Body Centered Cubic (BCC), and FCC (Face Centered Cubic) arrangements. Finite contact spacing's (2\% of particle diameter) are maintained between the spherical particles to avoid highly skewed elements during the grid generation process. Our results are compared with available experimental and numerical data for both continuum no-slip and also rarefied slip flows. For continuum no-slip flows, packing form of structured beds had a considerable influence on the pressure drop, particularly at higher Reynolds numbers. However, for rarefied flows, the influence of packing form seemed to be negligible. It was also observed that infinite random porous media correlations are unsuitable for predicting the friction factors and/or pressure drop in structured porous media for

\footnotetext{
* Corresponding author.

Email address: sridhar.palle@jsums.edu, saliabadi@jsums.edu
} 
continuum no-slip flows. Additionally rarefaction reduces the pressure drop and impacts the friction factor correlations. Due to lack of consistent generalized correlations in structured porous media and also since continuum correlations are not applicable for rarefied flows, new improved correlations for predicting friction factors in infinite structured microporous media are proposed.

KEYWORDS: Structured porous media, Knudsen number, rarefaction, micro/nano, friction factor, Hybrid FE/FV

\section{Introduction}

Porous media flows are abound in many different industrial and chemical processing applications and also in nature. Typical applications include packed bed generators, absorption towers, electronic cooling, transpiration cooling, catalytic bed reactors, filters, hydrogen storage, fuel cells, ground water hydrology, petroleum engineering, tight gas and shale gas reservoir systems, and soil mechanics. Porous media has been represented and studied in the past through either random beds or structured beds of particles. Random packed beds have been prevalent over 70 years in the industry mainly due to their low cost and ease of use [1]. Novel structured catalytic reactors [2] such as composite structured packing's have recently become popular due to their superior performance in terms of lower pressure drops. Structured beds have also been observed to have superior heat transfer efficiencies per pressure drop [3, 4]. Structured or regular beds have also been used in numerical studies as a simplification or first approximation for random beds. Whether it is random or structured, porous media geometries are highly complicated and pose a challenge for numerical studies in terms of grid generation and the computing power required.

Due to such highly complex geometries, majority of the earlier studies dealing with porous media applications were mostly based on empirical correlations (for higher Reynolds numbers), Darcy averaged flow models $\left(\operatorname{Re}_{d p}<1\right)$, and more recently lattice Boltzmann methods. Complexities further increase in rarefied micro and nano porous media with particle 
diameters approaching the mean free path of gas molecules. Particle diameters of porous walls in transpiration cooling and porous hydrogen storage alloys are typically in the range of 1-30 $\mu \mathrm{m}[5]$. Pore throat dimensions for shale gas and tight gas reservoir systems vary widely from $0.01 \mu \mathrm{m}$ to $50 \mu \mathrm{m}$. Mean free path of gaseous fuel transport through carbon paper in fuel cells and also in nanopores is comparable to the pore size [6].

When the mean free path of a gas becomes comparable to the characteristic lengths of the domain through which it is flowing, rarefaction effects dominate and gas slippage occurs. Knudsen number defines such degree of rarefaction for dilute gaseous flows and is defined as the ratio of the molecular mean free path $(\lambda)$ to the characteristic length $\left(L_{c}\right)$ such as the hydraulic pore diameter for porous media flows. For flows with very small Knudsen numbers $\left(K n \leq 10^{-3}\right)$ continuum approximation is valid, whereas for very large Knudsen numbers ( $K n$ $\geq 10$ ) continuum assumption completely breaks down and fluid flows in this regime are treated as free molecular flows. Rarefied gases typically encountered in micro and nano porous media fall in the slip $\left(10^{-3} \leq K n \leq 10^{-1}\right)$ and transition regimes $\left(10^{-1} \leq K n \leq 1\right)$. For nonsmall Knudsen number values $\left(K n>10^{-3}\right)$ no-slip boundary conditions are no longer valid and the numerical treatment of fluid flows in the slip flow regime $\left(10^{-3} \leq K n \leq 10^{-1}\right)$ typically involves the velocity slip modification for the boundary in the governing equations. Together with the mesh generation challenges in these highly complicated porous media geometries, slip boundary conditions further add to the complexity involved in direct pore level studies.

Previous studies which have dealt with slip flows in microporous media have mostly dealt with Klinkenberg effect which states that gas permeability differs significantly compared to liquids for the same porous geometry due to gas slippage. Such studies mostly focused on the influence of rarefaction on permeability at low velocities $\left(\operatorname{Re}_{d p}<1\right)$, and therefore were mostly based on volume averaged Darcy models. However, when gaseous velocities through porous media are higher such as in transpiration cooling, and sintered 
porous media heat exchangers, inertia effects cannot be neglected and warrant more direct pore-level analysis.

Advances in computational capacity and hardware have very recently enabled researchers to focus much more on such direct pore level simulations of fluid flow through porous media. Significant computational research has been conducted in the past for analyzing continuum flows in random porous media [8-19]. These studies focused on both infinite and also wall bounded random beds in detail using particle level simulations. Majority of the studies, agree with the famous Ergun correlation [7] in predicting the pressure drop in infinite random porous media. Eisfeld and Schnitzlein [10] conducted a detailed review of extensive experimental data and also existing correlations for evaluating the influence of walls on the pressure drop in random porous media. They found that Reichlet's [11] approach of correcting the Ergun equation for the wall effect was more promising and provided their own improved correlations for different random packing's of beds.

Even though a plethora of research exists on the random packed beds, structured beds have been gaining in popularity only recently. Part of the reason for more widespread use of random packed beds is primarily due to their low cost and ease of use even though they might not be the most efficient packing's compared to structured beds [1]. But with the increasing requirements of lower pressure drop and better overall heat transfer rates per pressure drop, structured packed beds have been receiving increasing interest in chemical and industrial applications $[1,2,20-25]$. These studies have identified that the Ergun correlation may not be suitable for predicting the friction factors in structured beds. Yang et al. [3, 4] particularly studied continuum flows in infinite structured porous media represented by spherical and ellipsoid particles arranged in different configurations including SC, BCC, and FCC packing arrangements. Based on their experimental and numerical studies, they have proposed correlations in predicting the friction factors for continuum flows in structured porous media. 
Literature on the numerical studies for understanding rarefaction influence in micro and nano porous media is even more limited due to the complexities arising from geometry creation, mesh generation, and also slip boundary modicifications. However, rarefied flows are routinely encountered in many porous media applications which are of significant practical interest such as transpiration cooling, fuel cells, sintered porous media heat exchangers, $\mathrm{CO}_{2}$ sequestrian into geological porous formations, gas production from coal beds, shale and tight gas reservoir systems [26]. To overcome the difficulties associated with complicated porous media geometries, few studies have recently addressed lattice Boltzmann methods $[6,27]$ in modeling rarefied flows. Accurate representation of rarefied fluid physics in these micro and nano porous systems is however highly important and if the computational challenges associated with mesh generation and performance can be addressed, direct particle level simulations can be much more reliable and accurate and therefore need to be further explored.

The work of $\mathrm{Xu}$ et al. [5] is one of the very few works that have conducted particle level simulations in addressing micro-pore scale effects in sintered microporous media. They studied rarefaction effects in infinite porous media represented by spherical particles in three different packing arrangements (SC, BCC, and FCC). Their studies were however limited to rarefied flows at very low Knudsen numbers $(0.0006$ to 0.01$)$ and also at very low Reynolds numbers. It is not known if these results are applicable over a wide range of Knudsen numbers and also at higher Reynolds numbers in the laminar flow regime. They validated their numerical results with experimental data including compressibility.

As the structured packed beds are gaining in popularity, it is highly imperative that accurate and robust correlations are available for predicting the pressure drop. Additionally, accurate prediction of the rarefaction influence is also highly critical in understanding the fluid physics in many of the practical micro and nano porous media systems. Therefore, the 
present work aims in studying both continuum and also rarefied slip flows in infinite structured porous media in detail through direct pore level simulations. Apart from their potential for direct chemical engineering applications, structured porous media may also be explored as a first approximation for more complicated soil mechanics and gas reservoir systems which typically involve random beds, particularly for rarefied flows.

In this work, we plan to validate hybrid Finite Element/Volume (FE/FV) methods for both macro-scale and also micro/nano porous media applications and to our knowledge, the present work is the first of its kind in extending hybrid FE/FV methods for studying rarefied flows in micro and nano porous media. The focus of the present work is in the continuum noslip $\left(K n<10^{-3}\right)$ and also rarefied slip flow regimes $\left(10^{-3}<K n<10^{-1}\right)$, where majority of micro/nano porous systems operate. In this work, infinite structured porous media is represented by spherical particles in three different packing arrangements; simple cubic (SC), body centered cubic (BCC), and face centered cubic (FCC).

\section{Problem Statement and Formulation}

Hybrid FE/FV methods have been extended for studying infinite structured micro porous media represented by sphere packing's. Our proprietary hybrid FE/FV flow solver which is based on a combination of finite element and finite volume methods is used to simulate fluid flow through the infinite structured porous media. Since simulating flow around hundreds of particles is a huge challenge, particles are cut into symmetric units and stacked into packed channels as shown in Fig.1 for all three packing arrangements (SC, BCC, and FCC). Flow is considered along the length of the channel in the x-direction. The geometry and flow domain details are summarized in detail in Table 1 for the three packing arrangements. 
SC
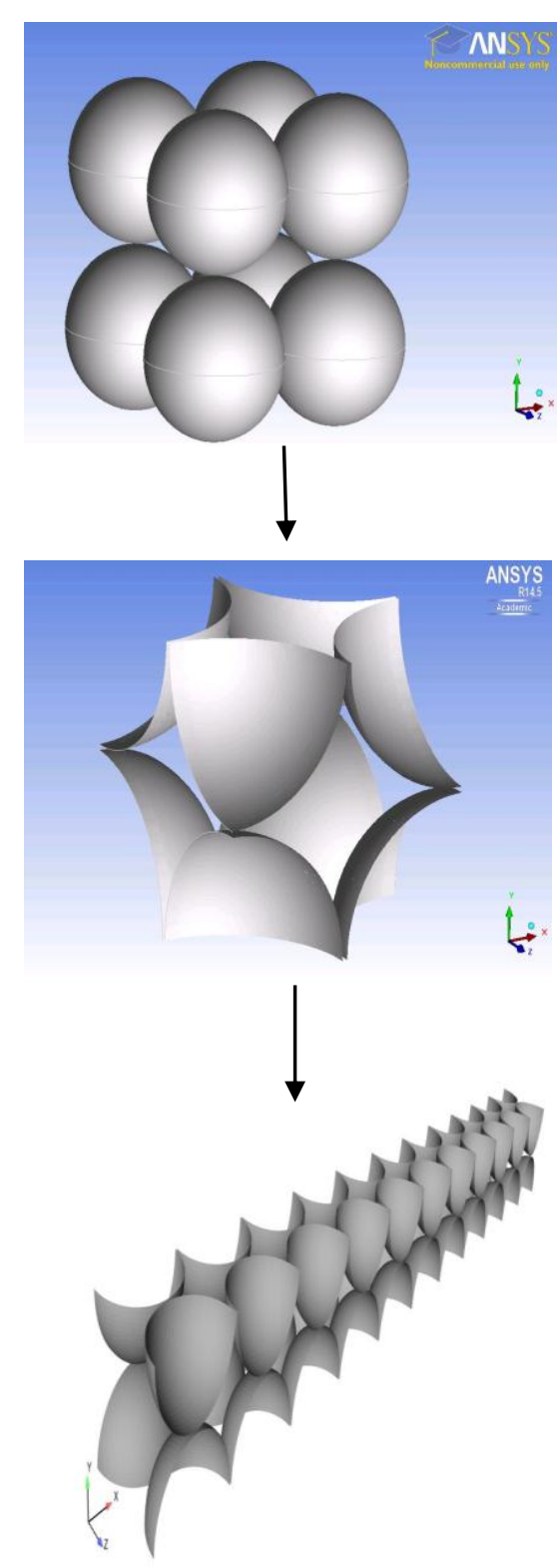

(a)
BCC
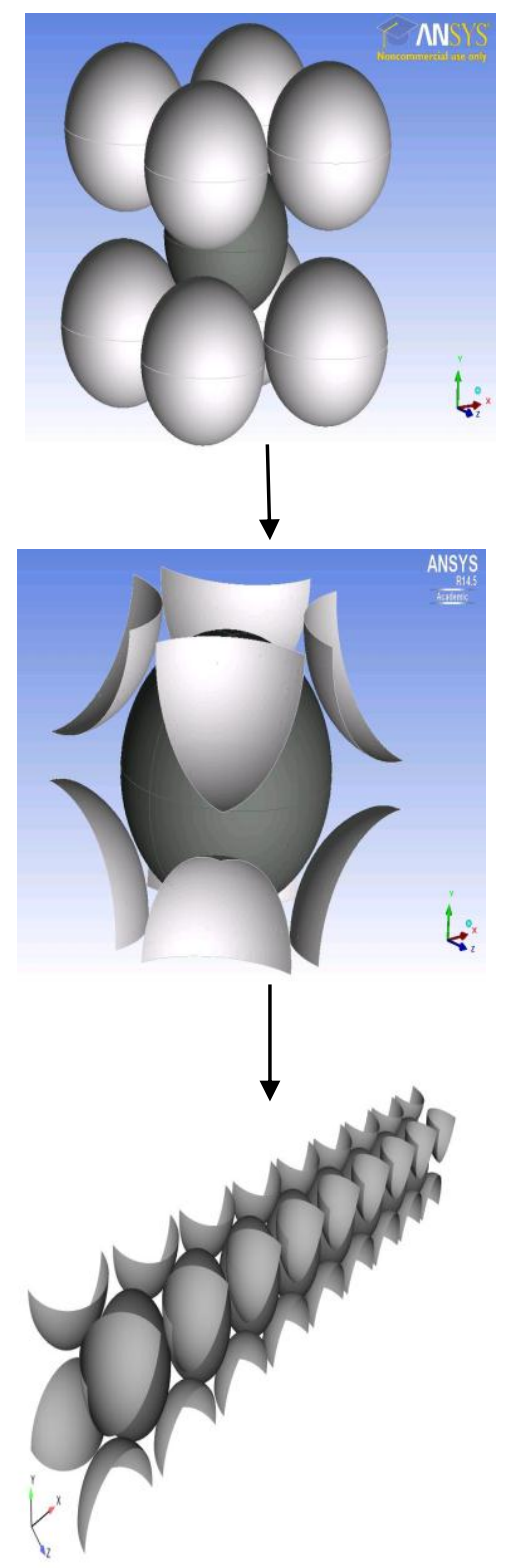

(b)
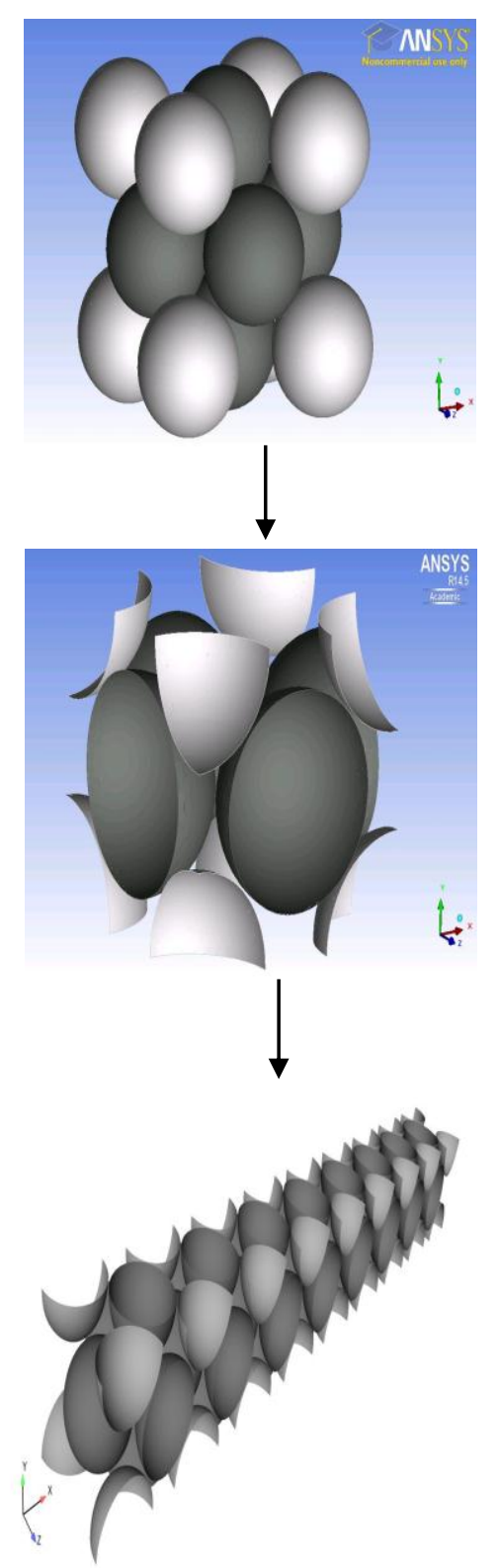

(c)

Figure 1: Infinite Structured Porous Media (a) SC, (b) BCC, and (c) FCC

\section{Table 1}

Geometry and grid parameters

\begin{tabular}{llllll}
\hline $\begin{array}{l}\text { Packing } \\
\text { Form }\end{array}$ & Porosity & $\begin{array}{l}\text { Extended } \\
\text { Entrance Length }\end{array}$ & $\begin{array}{l}\text { Porous Bed } \\
\text { Length }\end{array}$ & $\begin{array}{l}\text { Extended } \\
\text { Exit Length }\end{array}$ & Grid Size \\
\hline SC & 0.5 & $5 d_{p}$ & $11.2 d_{p}$ & $8 d_{p}$ & 3.0 Million \\
BCC & 0.37 & $5 d_{p}$ & $10.44 d_{p}$ & $8 d_{p}$ & 3.1 Million \\
FCC & 0.3 & $5 d_{p}$ & $12.544 d_{p}$ & $8 d_{p}$ & 5.7 Million \\
\hline
\end{tabular}


The flow domain includes an extended entrance region $\left(5 d_{p}\right)$ and an extended exit region (8 $d_{p}$ ) (not shown in Fig.1). Symmetry boundary conditions are assumed on all sides (top, bottom, left, and right) for the extended entrance, packed bed, and extended exit regions. Uniform velocity is assumed at the extended inlet and outflow boundary conditions are considered at the extended channel exit. For continuum simulations no-slip conditions are maintained at the sphere surfaces and for rarefied simulations, velocity slip as defined by Maxwell [28] is considered at the sphere walls.

$u_{g a s}-u_{w}=\frac{2-\sigma_{v}}{\sigma_{v}} K n_{d p}\left(\frac{\partial u}{\partial n}\right)_{w}$

where $(\partial u / \partial n)_{w}$ is the velocity gradient normal to the sphere surface. $\sigma_{v}$ is the tangential momentum accommodation coefficient, which is the property of gas-wall surface interaction and is dependent on the composition, temperature, and velocity of gas on the surface, and also on the wall surface temperature, chemical state, and roughness [29, 34]. Typically it is assumed to be unity for many practical engineering applications with rough surfaces $[29,34]$. Therefore, in the present study $\sigma_{v}$ was assumed to be unity. For highly polished surfaces however, $\sigma_{v}$ can range from 0.8 to $1[34]$, The effect of any sub-unity momentum accommodation coefficients would be to further enhance the Knudsen number and slip effects for a given gas-surface combination. Non-dimensional governing equations describing the incompressible flow through structured beds are listed below:

$\tilde{\mathrm{N}} \times \mathbf{u}=0$,

$\frac{\mathbb{q} \mathbf{u}}{\mathbb{\Phi} t}+\mathbf{u} \times \nabla \mathbf{u}=\nabla \times \boldsymbol{\sigma}$

$\boldsymbol{\sigma}=-\mathrm{p} \mathbf{I}+\frac{2}{R e_{d p}} E(\mathbf{u}) ; \quad \mathbf{E}=\frac{1}{2}\left(\nabla \mathbf{u}+\nabla \mathbf{u}^{\mathrm{T}}\right)$,

The strain tensor is denoted by $\mathbf{E}$ and $\mathbf{I}$ represents the identity tensor. For turbulent flows $\left(\operatorname{Re}_{d p}>300\right)$, we use one-equation Spalart-Allmaras DES model [30] which is derived from 
the Spalart-Allmaras one-equation eddy-viscosity RANS model. The equation can be written as shown below:

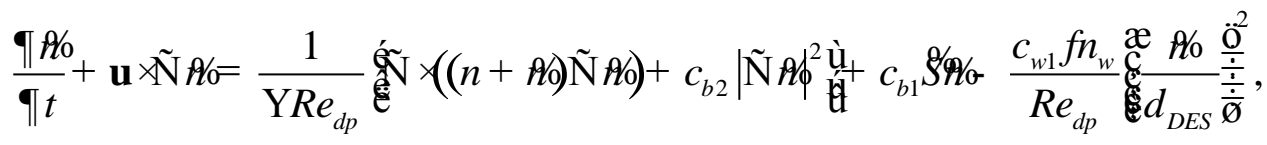

where $A_{c}$ is the working variable in the order of the molecular viscosity $n$. If the molecular viscosity is constant, then $n=1 \mathrm{In} \mathrm{Eq.} \mathrm{(5),} \mathrm{the} \mathrm{second} \mathrm{and} \mathrm{the} \mathrm{last} \mathrm{term} \mathrm{on} \mathrm{the} \mathrm{right} \mathrm{hand} \mathrm{side}$ are the production term and destruction term, respectively. For more details about the implementation of DES model see [31-33].

\section{1. $\quad$ Pressure drop and Friction Factor Terminology}

Non-dimensional pressure drop, friction factors, and Reynolds numbers in packed bed studies are represented in different ways which could some time lead to confusion. Recently Palle and Aliabadi [25] described in detail different forms of expressions in literature for hydraulic diameters, friction factors, and Reynolds numbers for porous media. Hydraulic diameter $\left(d_{h}\right)$ for porous media which is sometimes also called hydraulic radius is defined as follows:

$d_{h}=\frac{\varepsilon d_{p}}{(1-\varepsilon)}$

where $\varepsilon$ is the porosity. Reynolds number is defined in two ways; particle Reynolds number $\left(R e_{d p}\right)$ and also Reynolds number based on hydraulic diameter $\left(R e_{E}\right)$ :

$$
\operatorname{Re}_{d p}=\frac{\rho U_{s} d_{p}}{\mu}, \operatorname{Re}_{E}=\frac{\rho U_{0} d_{h}}{\mu}=\frac{\operatorname{Re}_{d p}}{(1-\varepsilon)}
$$

where superficial velocity $\left(U_{s}\right)$ and the average fluid velocity $\left(U_{0}\right)$ inside the pores, are defined as follows:

$U_{s}=\frac{Q}{A_{T}}, \quad U_{o}=\frac{Q}{A}=\frac{U_{s}}{\varepsilon}$, 
where $Q$ is the volumetric flow rate, $A_{T}$ is the total cross-sectional area of the packed bed, and $A$ is the cross-sectional area available for the flow. Similarly Knudsen number is also defined in terms of particle diameter $\left(d_{p}\right)$ and also hydraulic diameter $\left(d_{h}\right)$

$$
K n_{d p}=\frac{\lambda}{d_{p}}, K n_{d h}=\frac{\lambda}{d_{h}}=\frac{(1-\varepsilon)}{\varepsilon} K n_{d p}
$$

where $\lambda$ is the mean free path of gas flowing through the porous media.

The well known correlation for pressure drop across packed beds is of Ergun [7]:

$$
\frac{\Delta P}{L}=A_{E} \frac{(1-\varepsilon)^{2}}{\varepsilon^{3}} \frac{\mu U_{s}}{d_{p}^{2}}+B_{E} \frac{(1-\varepsilon)}{\varepsilon^{3}} \frac{\rho U_{s}^{2}}{d_{p}},
$$

Where $A_{E}$ and $B_{E}$ are parameters for evaluating the pressure drop which for Ergun relation are listed as $A_{E}=150$ and $B_{E}=1.75$. Rearranging the above equation one can obtain dimensionless pressure drop and/or friction factor correlation as shown below:

$$
\begin{aligned}
& \frac{\Delta P}{\rho\left(U_{s}\right)^{2}} \frac{d_{p}}{L} \frac{\varepsilon^{3}}{(1-\varepsilon)}=\frac{\Delta P}{\rho\left(U_{s} / \varepsilon\right)^{2} L} \frac{\varepsilon d_{p}}{(1-\varepsilon)}=f_{E}=A_{E} \frac{(1-\varepsilon)}{\operatorname{Re}_{d p}}+B_{E} . \\
& f_{E}=\frac{A_{E}}{\operatorname{Re}_{E}}+B_{E}
\end{aligned}
$$

\section{Numerical Method}

The incompressible governing equations are solved using our recently developed matrix free, fully parallelized, implicit, hybrid finite element/finite volume (FE/FV) flow solver which is based on a pressure correction or projection method. We use the cell-centered finite volume method (FV) to solve the momentum equation for the intermediate velocity and the nodebased Galerkin finite element method (FE) to solve the Poisson equation for the pressure correction. Finite volume methods are better suited for handling high aspect ratio elements typically found in boundary layers, whereas Finite elements methods are more suitable for the elliptic type of equations such as Poisson equation. Therefore to take advantage of the merits 
of both the methods, while avoiding their shortcomings, finite volume method is used to solve the momentum and turbulence equations and finite element method is used for solving the pressure Poisson equation. Note that in our implementation, the pressure does not directly enter the momentum equation. Instead, an auxiliary variable closely related to the pressure takes the place of pressure in the momentum equations. The auxiliary variable updates the pressure and velocity fields. Because the velocity components and the auxiliary variable are placed at different locations on the mesh, the current hybrid scheme can be viewed as a staggered-mesh scheme. However, our staggered mesh deployment is distinct from the more traditional staggered mesh scheme where the pressure is put on the cell center and the velocity is put on the cell interfaces. In our deployment, the velocity unknowns are put at the cell centers and the auxiliary variable is put at the mesh vertices. This deployment makes it convenient to compute the gradients of the auxiliary variable using local finite element basis function, which is required in solving the momentum equations. Numerical experiments show that the current staggered deployment also avoids the notorious velocity-pressure odd-even decoupling phenomenon. Another key feature of the current hybrid scheme is that it allows the same order of spatial discretization for velocity and pressure. In other words, the velocity takes a second-order accurate reconstruction in the FV framework and the pressure uses linear basis functions in the FE framework. The pressure is updated carefully to ensure it is free of any unphysical boundary layer. Previous numerical results have shown that our hybrid implementation is superconvergent in terms of the spatial convergence rates for both velocity and pressure [31-33]. More details regarding the implementation of finite element/volume methodology can be found in our previous works [31-33].

\section{Results and Discussion}

Both no-slip and also rarefied slip flows are numerically simulated using our hybrid FE/FV flow solver in infinite structured porous media represented by spherical particles in SC, BCC, 
and FCC packing arrangements. Table 1 lists the geometry, flow domain, and porosity details for the three packing arrangements. Flow can be treated as continuum for macro-scale porous media applications and also for micro porous media when Knudsen numbers are very low $(K n<0.001)$. For such continuum flows, Reynolds numbers can vary over a wide range, and therefore we have conducted numerical simulations for different packing's of structured beds for $1 \leq R e_{E} \leq 4000$ when analyzing no-slip flows. However, for rarefied flows with higher $K n$ values, particle diameters $(0.001$ to $30 \mu \mathrm{m})$ and subsequently Reynolds numbers are typically very low in micro/nano porous media. Therefore only laminar flows in the range of $R e_{E}=1$ to 300 are numerically investigated for potential rarefaction effects in different structured packing arrangements. For majority of practical engineering applications with rough surfaces, momentum accommodation coefficients $\left(\sigma_{v}\right)$ were found to be near unity $[29,34]$. Therefore, in the present study, $\sigma_{v}$ was chosen to be unity.

\subsection{Grid Independence}

Grid independence and validation studies of our hybrid FE/FV flow solver for structured porous media flows were conducted in detail for wall bounded Simple Cubic (SC) beds in the earlier work of Palle and Aliabadi [25]. Nevertheless, for the sake of completeness and also since infinite structured beds in different packing arrangements are studied in this work, additional grid independence studies are conducted and are discussed below. For avoiding highly skewed mesh elements, finite contact spacing's $\left(2 \% d_{p}\right)$ were allowed between the porous particles. In the past, several studies $[1,15,25]$ allowed finite contact spacing's in direct porous media simulations and observed very small differences due to finite contact spacing's. This can also be seen from Fig.2 (a) for infinite SC beds, where friction factor has been plotted as a function of Reynolds numbers at $0 \% d_{p}$ and $2 \% d_{p}$ contact spacing's. Allowing for finite contact spacing's between the spheres influences the friction factors, but only marginally compared to the no-contact spacing's results. 


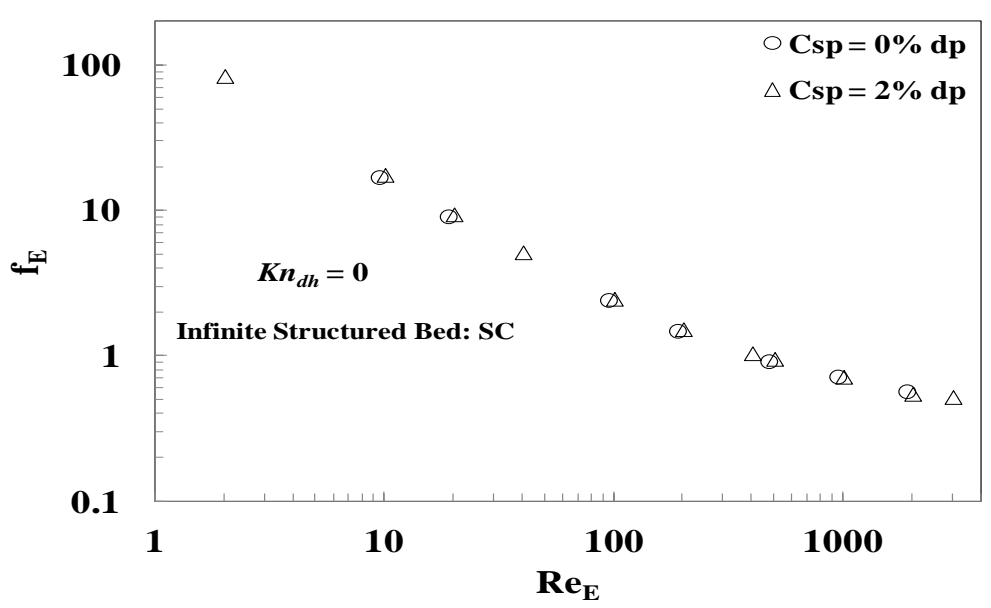

(a)

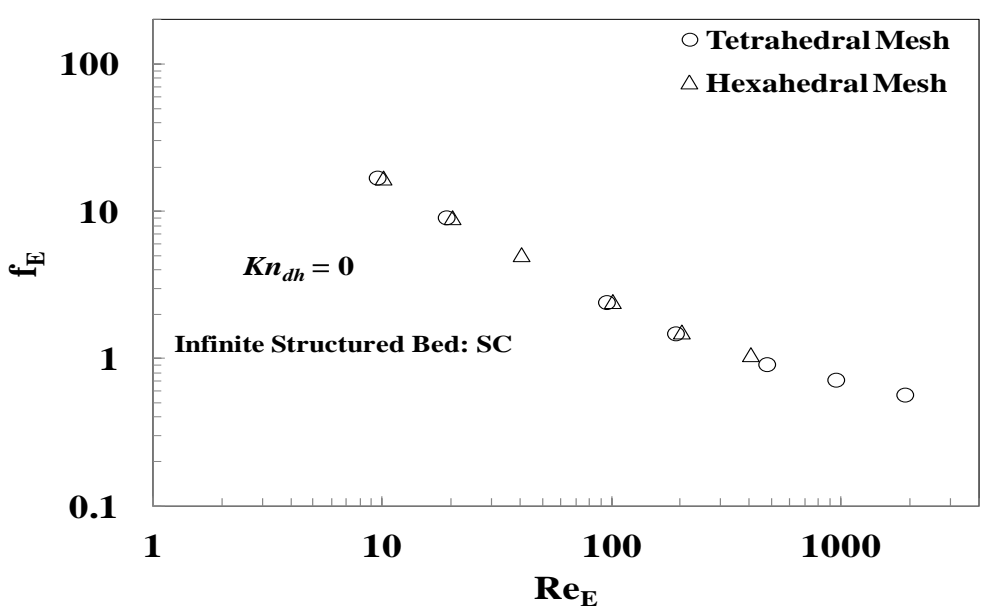

(b)

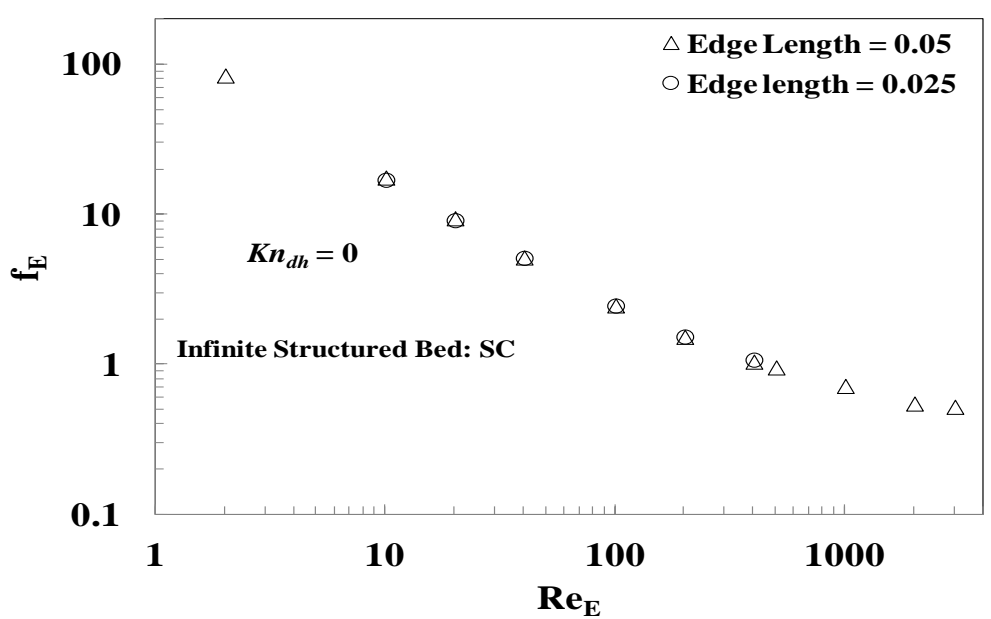

(c)

Figure 2: Friction factors for infinite SC Beds. (a) Influence of contact spacing between spheres, (b) Tetrahedral and hexahedral mesh comparison, and (c) Grid Independence 
We have also tested both tetrahedral and hexahedral mesh element types for SC beds, and it can be seen from Fig. 2(b), that results from both types of meshes are in good agreement. Figure 2(c) shows the grid independence for SC beds at two different mesh element edge lengths $(\Delta s=0.05$, and 0.025). All further results presented and discussed are based on tetrahedral mesh elements with an edge length of $\Delta s=0.025$ for all three packing arrangements. Since rarefied flows are also being studied which need finer mesh elements near the boundary, prismatic elements were used at the sphere walls, with first layer thickness of 0.0015 , and with a growth rate of 1.2. Total grid elements for the three packing's of infinite structure beds are listed in Table 1.

\subsection{Continuum No-Slip Flows}

Continuum no-slip flows $(K n \leq 0.001)$ are first discussed to analyze the influence of packing arrangement on fluid velocity and pressure drop. For macro-scale porous media and even for microporous flows, when the mean free path of gas is considerably smaller compared to the porous particle diameter, fluid can still be treated with no-slip conditions. For continuum flows, Reynolds numbers can vary over a wide range. Therefore both laminar and turbulent flows are analyzed by varying the Reynolds numbers from $1 \leq \operatorname{Re}_{E} \leq 4000$.

\subsubsection{Streamlines}

Non-dimensional velocity streamlines for the three packing forms of infinite structured beds are plotted in Figs. 3-5 at two different Reynolds numbers $\left(\operatorname{Re}_{d p}=10\right.$ and $\left.\operatorname{Re}_{d p}=100\right)$. From these figures it can be seen that maximum velocities are highest for FCC and BCC beds compared to SC beds. It can also be seen that tortuosity is highest for FCC beds, followed by BCC, and SC beds. At lower Reynolds numbers, the fluid flow is laminar, and sphere wall surface friction dominates. However, as the Reynolds number increases, inertia effects are much more dominant, and the tortuous a path the fluid has to take might add to the pressure 
drop. The influence of packing form on infinite structured beds can be understood clearly by looking at the non-dimensional pressure drop and friction factors in the next section.

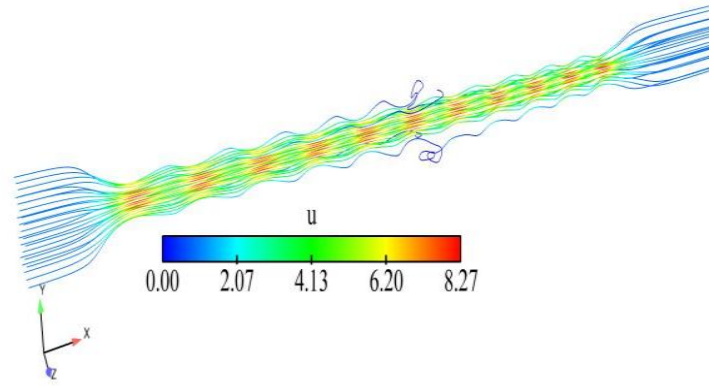

(a)

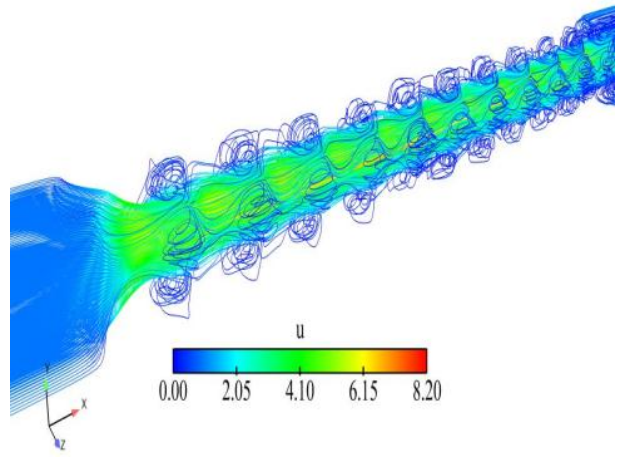

(b)

Figure 3: Streamlines for infinite SC Beds (a) $R e_{d p}=10$ and (b) $R e_{d p}=100$

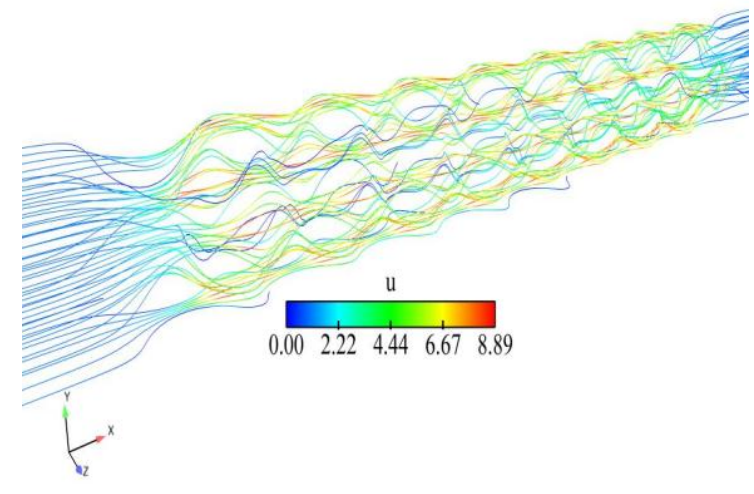

(a)

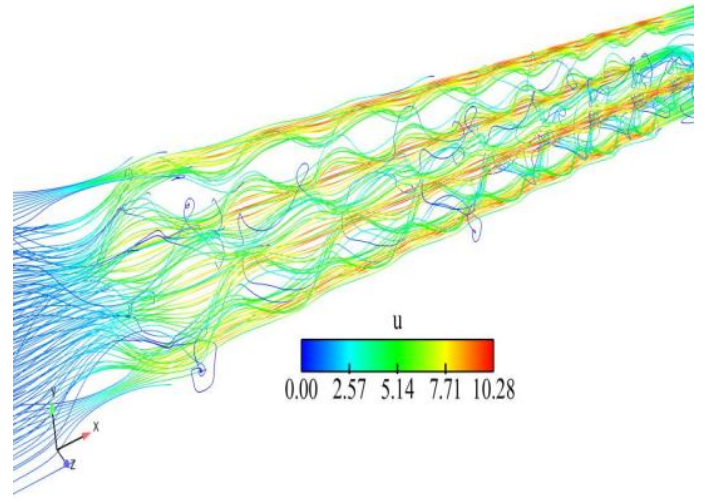

(b)

Figure 4: Streamlines for infinite BCC Beds (a) $\operatorname{Re}_{d p}=10$ and (b) $\operatorname{Re}_{d p}=100$

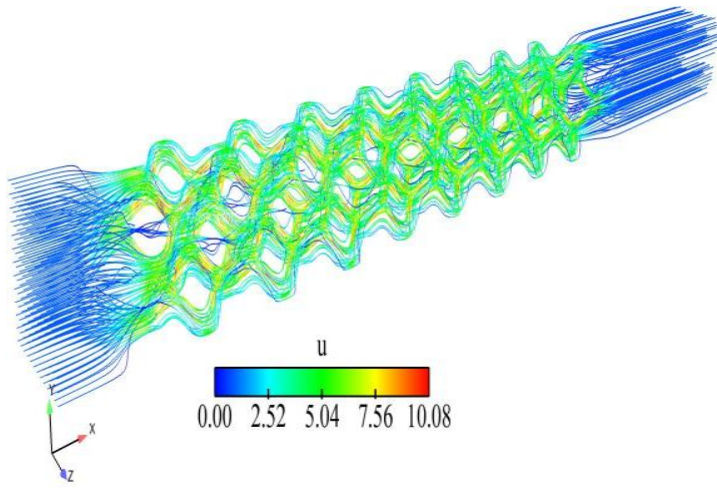

(a)

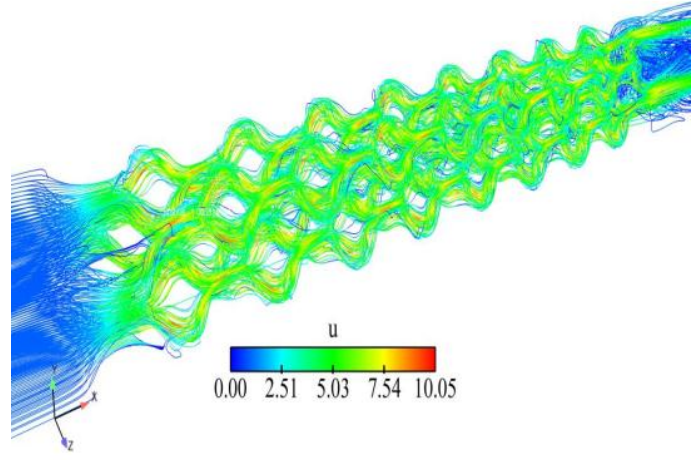

(b)

Figure 5: Streamlines for infinite FCC Beds (a) $R e_{d p}=10$ and (b) $R e_{d p}=100$ 


\subsubsection{Friction factors}

Non-dimensional pressure drop can be rearranged as shown in Eq. (11), to account for the bed porosity and the resulting pore-scale friction factors are plotted in Fig. 6 for the three packing's of infinite structured beds. Our results are compared with previously published numerical and experimental data of Yang et al [3, 4] and also the Ergun correlation [7] which is typically used to predict the pressure drop in infinite random beds as shown below in Eq. (13).

$f_{E}=\frac{A_{E}}{\operatorname{Re}_{E}}+B_{E}, \quad A_{E}=150, \quad B_{E}=1.75$

One observation obvious from these figures is that, Ergun equation clearly over predicts the friction factors for all three packing forms at higher Reynolds numbers. This is because of the channeling effect present in the structure beds and absent in the random beds for which the Ergun equation is applicable. At very low Reynolds numbers $\left(R e_{E}<20\right)$, flow is laminar and viscous effects and sphere surface friction dominate and there is not much difference between different forms of packing and Ergun equation can still be used for structured beds at this very low $\operatorname{Re}_{E}$ values. However, as the fluid velocity becomes higher, inertial effects dominate, and due to the increasing channeling effect and less tortuosity in structured beds compared to random beds, friction factors are lower. However within structured beds, tortuosity is lower for SC beds compared to BCC and FCC beds. For FCC beds fluid path is more tortuous and while channeling still present, friction factors tend to be closer to the random beds (represented by the Ergun correlation) as shown in Fig.6 (c).

We have also compared our numerical results for all three packing forms with available experimental and numerical data. As shown in Fig. 6(a), it can be observed that our results are in very good agreement with the computational and experimental data of Yang et al. [3, 4] for $R e_{E}<1000$ for SC beds. 


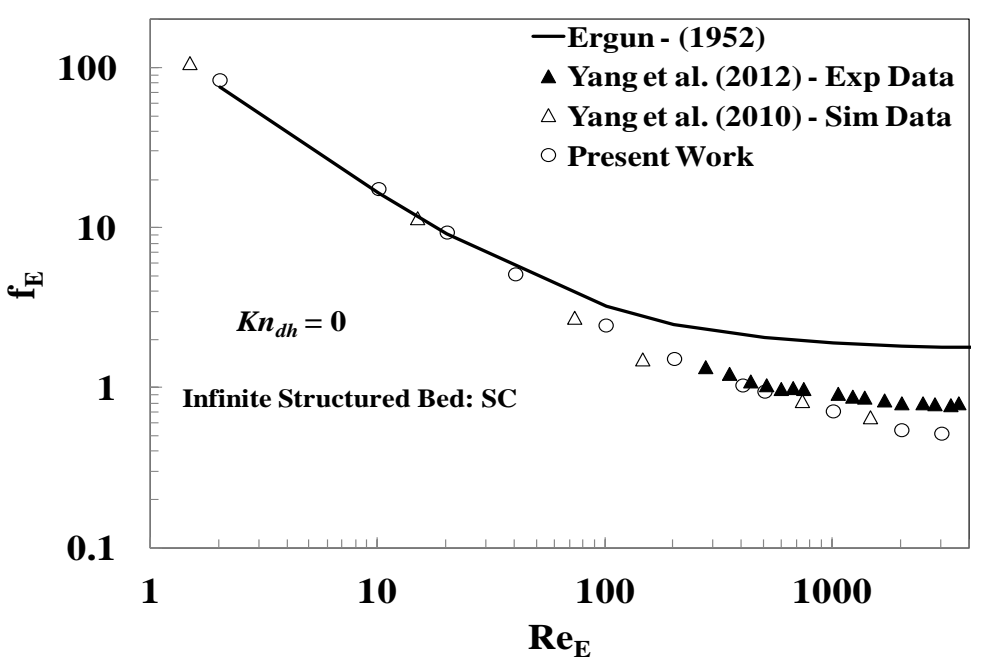

(a)

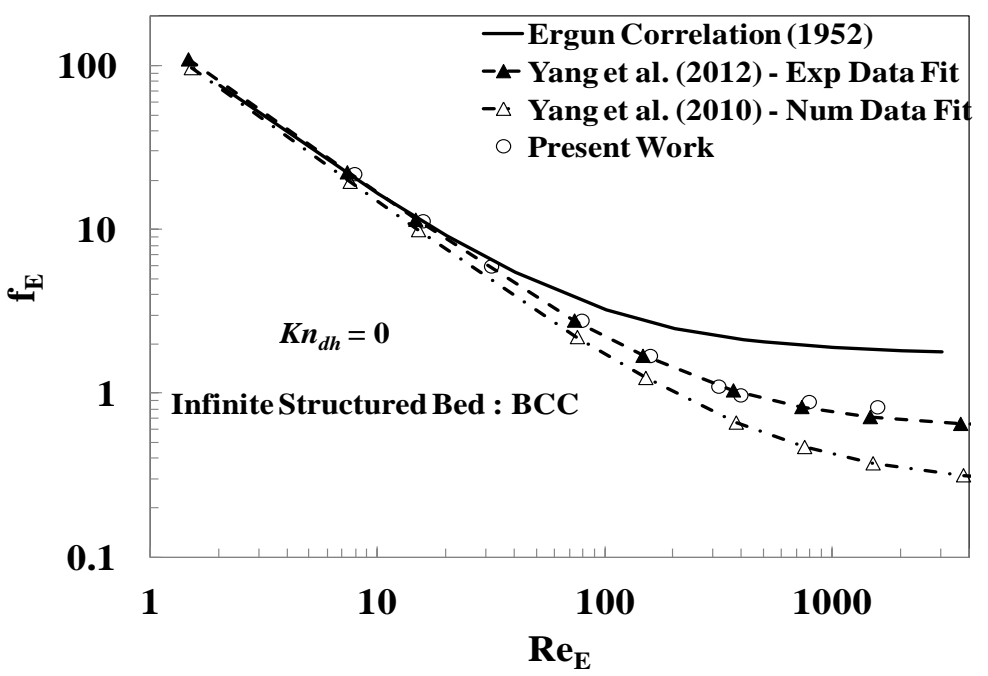

(b)

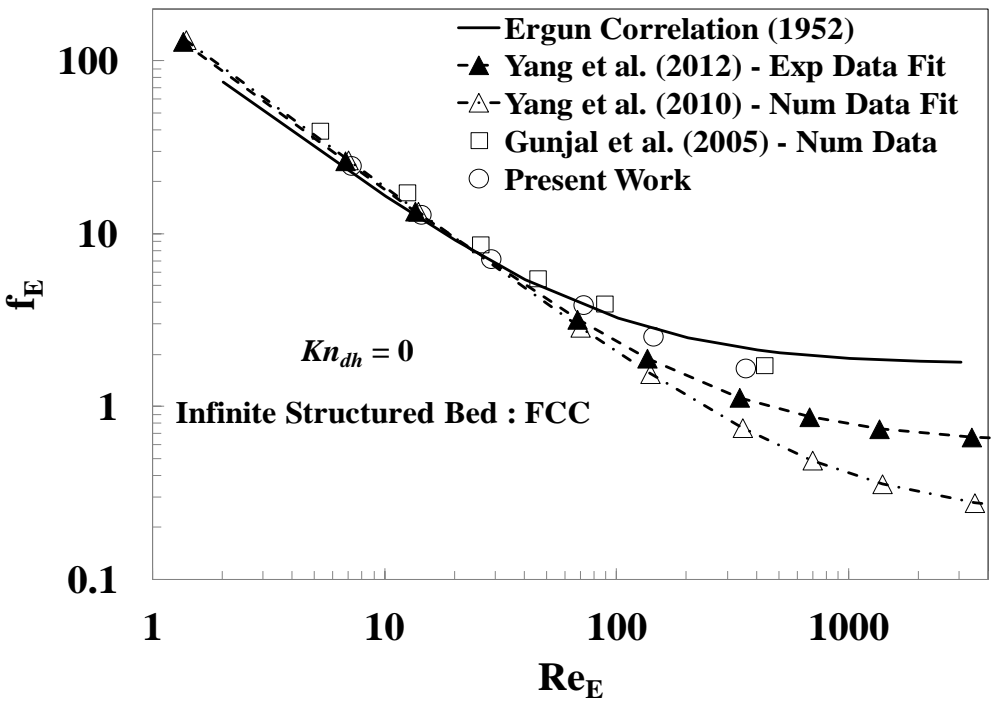

(c)

Figure 6: Friction factors for infinite structured beds (a) SC, (b) BCC, and (c) FCC 
At much higher Reynolds numbers $\left(R e_{E}>1000\right)$, our results are still in good agreement with their computational data, but are slightly under predicted compared to their experimental data. This slight discrepancy was explained in our previous work [25] dealing with wall effects in SC beds. On analyzing the experimental data of Yang et al. [4], their experimental set up for infinite SC beds had a channel hydraulic diameter-to-particle diameter ratio of $D_{h} / d_{p} \sim 6$. As has been documented in our previous study [25], wall effects are still significant at this ratio and the packed beds cannot be treated as infinite beds.

Our results for friction factors in infinite BCC beds plotted in Fig. 6(b) are in excellent agreement with the experimental data fit of Yang et al. [4] compared to their numerical data fit [3] which is under predicted. For FCC beds, as shown in Fig. 6(c), our results are closer to the experimental data of Yang et al. [4], but are over predicted in the turbulent flow regime, while their numerical data [3] is still very under predicted compared to their experimental data. Finite contact spacing's between sphere particles in our numerical data could account for some discrepancy with their experimental data, but the better explanation however could be through their channel hydraulic diameter ratio's which are $D_{h} / d_{p} \sim 6.39$ and $D_{h} / d_{p} \sim 7.53$ respectively for BCC and FCC beds. As explained before and observed in Fig.6 (a) to Fig.6(c), from SC to FCC beds, the fluid path becomes more tortuous and the friction factors for FCC beds trend towards the Ergun correlation (applicable for random beds) in turbulent flow regimes. For wall bounded random beds it has been shown previously [10] that, channel walls increase the friction factors in the laminar flow regime, but tend to decrease the friction factors in turbulent flow regime when compared to infinite beds. FCC beds, tend to approach random beds, and since the experimental data of Yang et al. [4] had finite wall effects $\left(D_{h} / d_{p} \sim 7.53\right)$, the friction factors might have been lower than truly infinite FCC beds as predicted in the present work. This is however, one of the possible explanations, while sphere wall contact spacing's, experimental uncertainty could also be 
other reasons for the discrepancy. For additional validation, we compared with the numerical data of Gunjal et al. [22] for friction factors in infinite FCC beds. As can be seen from Fig.6 (c), our results are in very good agreement with the data of Gunjal et al. [22] whose friction factors are also higher than the experimental data fits of Yang et al. [4]. The discrepancy within the numerical and experimental data fits of Yang et al. [3, 4] particularly in the turbulent flow regime was attributed by the authors $[3,4]$ to the finite contact spacing's between the particles in their numerical studies. However, the discrepancy was not small enough to be explained solely based on the finite contact spacing's which were observed to influence the friction factors marginally $[1,15,25]$.

Packing arrangement influence on the friction factors can be seen more clearly in Fig. (7) for continuum flows. Friction factors for SC and BCC beds follow closely in the laminar regime, but in the turbulent regime at higher Reynolds numbers, friction factors for BCC beds are slightly higher than SC Beds. Correspondingly friction factors for FCC beds are much higher than $\mathrm{BCC}$ beds and approach Ergun correlation prediction for random beds as explained earlier.

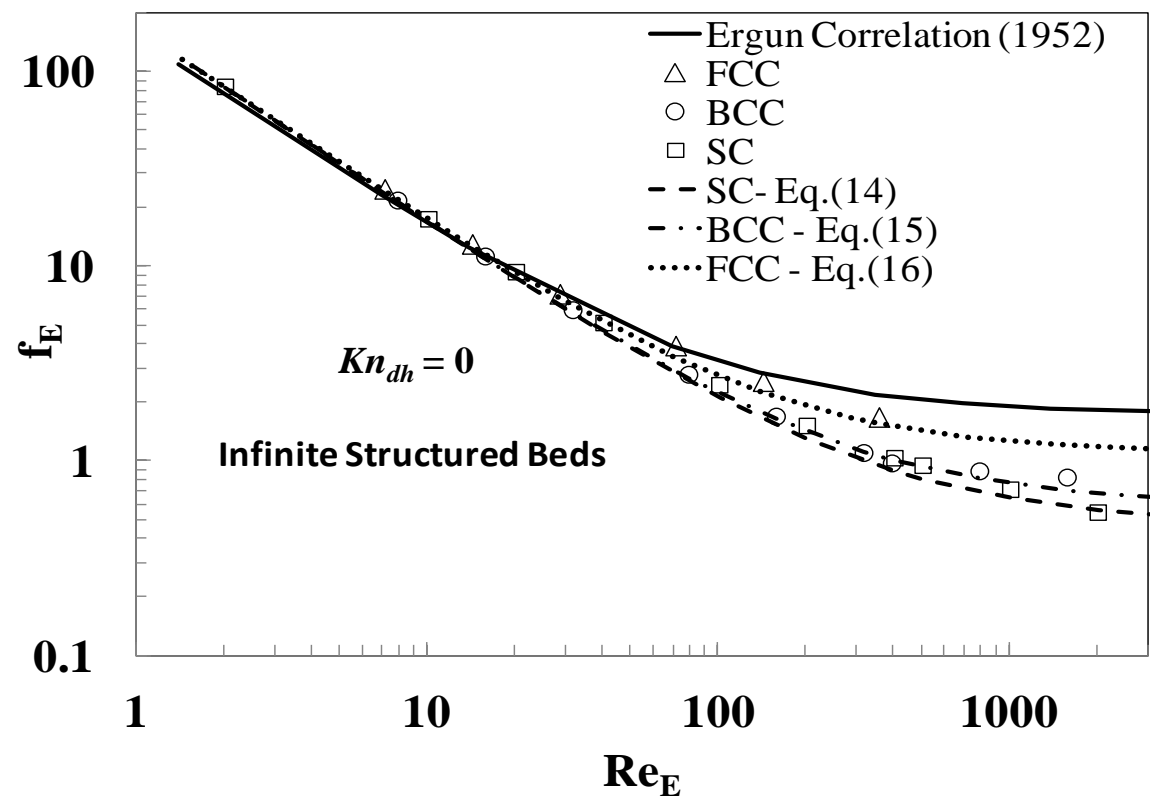

Figure 7: Packing arrangement influence on infinite structured bed friction factors 
For $R e_{E}<100$, Ergun correlation represents the pressure drop in FCC beds fairly accurately, but fails beyond that, because channeling effect is still present in the FCC beds, which becomes significant in the turbulent flow regime and results in lower friction factor values. Since accurate correlations for representing infinite structured beds are absent in the literature, we have curve fitted our numerical data and propose improved correlations for predicting the friction factors in infinite structured beds for continuum flows as shown below in Eq.'s (14)-(16) and also plotted in Fig. (7). Table 2 lists the parameters $A_{E}$ and $B_{E}$ for calculating pore-scale friction factors for different packing forms from our study in comparison with previous studies.

SC Beds: $\quad f_{E}=\frac{A_{E}}{\operatorname{Re}_{E}}+B_{E}, \quad A_{E}=165, \quad B_{E}=0.48$

BCC Beds: $\quad f_{E}=\frac{A_{E}}{\operatorname{Re}_{E}}+B_{E}, \quad A_{E}=165, \quad B_{E}=0.6$

FCC Beds: $\quad f_{E}=\frac{A_{E}}{\operatorname{Re}_{E}}+B_{E}, \quad A_{E}=165, \quad B_{E}=1.1$

\subsection{Influence of Rarefaction}

Results discussed above are for continuum no-slip flows applicable for both macro-scale porous media and also for micro-scale porous media where particle diameters are significantly higher compared to the mean free path of gas flowing through the porous media $(K n<0.001)$. However, in several micro/nano porous media studies such as in transpiration cooling and gas reservoir systems, Knudsen number values are much higher and fluid flow in these applications needs to be analyzed for potential rarefaction effects and gas slippage. Rarefied flow physics is completely different from continuum no-slip flows and the existing correlations for predicting pressure drop in continuum flows are also inapplicable.

In this study, therefore we have thoroughly analyzed rarefied flows in microporous media represented through different forms of structured packing's (SC, BCC, and FCC) in 
the slip flow regime $(0.001<K n<0.1)$ which has several practical micro/nano scale porous media applications. Since Reynolds numbers are typically very low for rarefied flows in micro and nanoporous media, only laminar flows are considered over $1<R e_{E}<300$.

\subsubsection{Velocity Contours}

For rarefied flows, no-slip conditions are no longer valid and fluid slips along the porous surfaces. Non-dimensional slip velocity on the sphere surfaces for SC beds in shown in Fig. 8 for different Knudsen number values. It can be seen that as the $K n_{d h}$ increases, slip velocity on the sphere particle surface also increases. Increase in the velocity near sphere wall surface due to slippage, is also accompanied by a reduction in the core velocities in the center of the channel. This can also be seen from Fig. 9 where z-mid plane velocities are plotted for SC beds at three different Knudsen number values. Maximum velocity occurs in the core of the channel, and decreases as rarefaction increases. Similar behavior can also be seen for BCC and FCC beds in Fig10 and Fig.11 respectively, where z-sectional plane velocities are plotted at different Knudsen numbers. Maximum core velocities reduce and velocities near sphere surface increase as rarefaction is increased. Increase in the sphere surface slip velocities and a reduction in the inner core velocities result in smaller velocity gradients near the sphere surface which will in turn impact the friction factors as discussed in the next section.

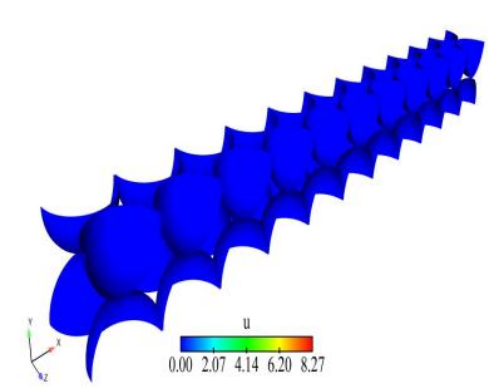

(a)

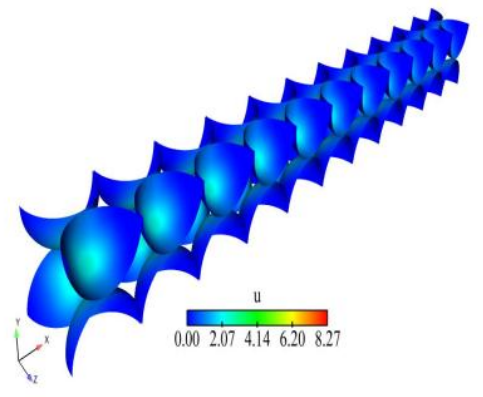

(b)

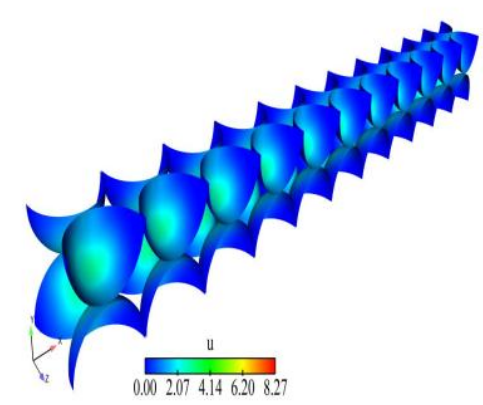

(c)

Figure 8: Slip velocity for SC beds (a) $K n_{d h}=0$, (b) $K n_{d h}=0.05$, (c) $K n_{d h}=0.1$ 


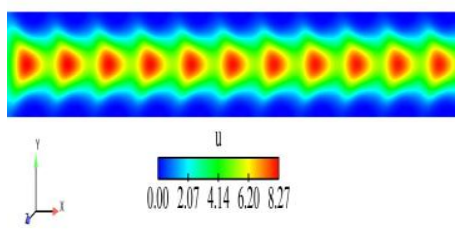

(a)

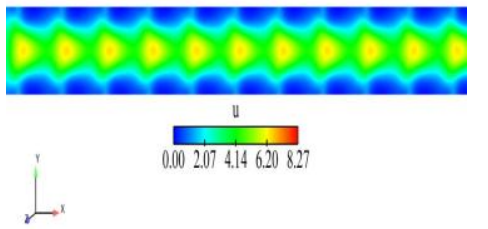

(b)

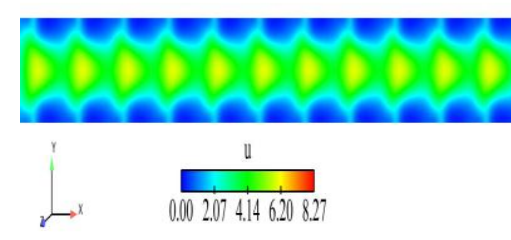

(c)

Figure 9: Z-midplane velocity for SC Beds (a) $K n_{d h}=0$, (b) $K n_{d h}=0.05$, (c) $K n_{d h}=0.1$

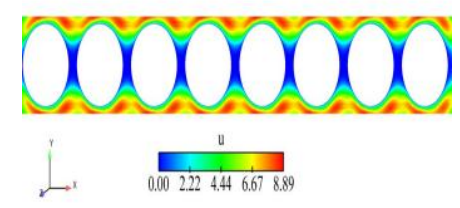

(a)

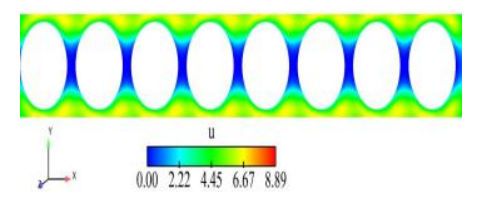

(b)

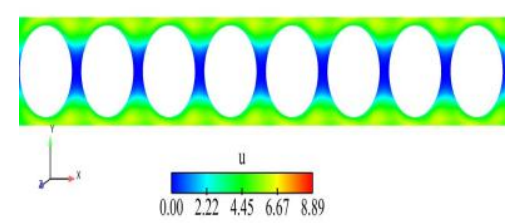

(c)

Figure 10: Z-midplane velocity for BCC Beds (a) $K n_{d h}=0$, (b) $K n_{d h}=0.05$, (c) $K n_{d h}=0.1$
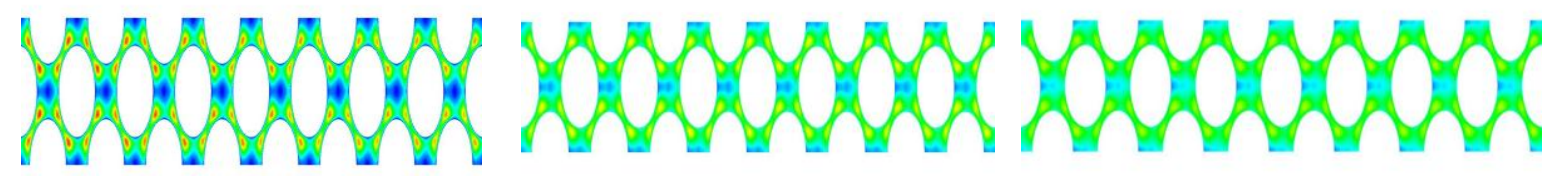

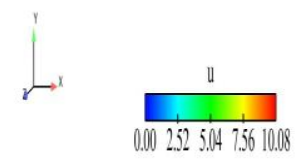

(a)

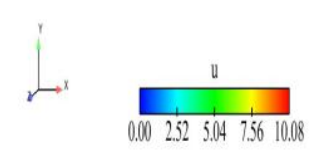

(b)

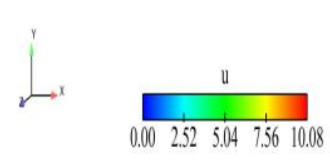

(c)

Figure 11: Z-plane velocity for FCC Beds (a) $K n_{d h}=0$, (b) $K n_{d h}=0.05$, (c) $K n_{d h}=0.1$ 


\subsubsection{Friction factors}

Our results for friction factors in rarefied flows are first compared with previously published numerical data of $\mathrm{Xu}$ et al. [5], who studied rarefied flows in sintered porous media with porous particle diameters varying from $10 \mu \mathrm{m}$ to $200 \mu \mathrm{m}$. Their study however was limited to low Knudsen numbers $\left(0.0006 \leq K n_{d h} \leq 0.01\right)$. Fig. 12 plots the friction factors from our study in comparison to the numerical data of $\mathrm{Xu}$ et al. [5] at an average Knudsen number of 0.0033. Our results, while slightly are over predicted by around $16 \%$ at lowest Reynolds number, are still in good agreement with their results at higher Re values. Their studies are based on sintered porous media, where particle surfaces overlap on each other, and our studies are based on finite contact spacing's $\left(2 \% d_{p}\right)$ between spheres. Additionally the mesh elements in their numerically study didn't incorporate any finer prismatic elements near the sphere surface boundary, which are crucial for more accurate evaluation of slip velocity across the sphere surfaces and may possibly explain the slight discrepancy.

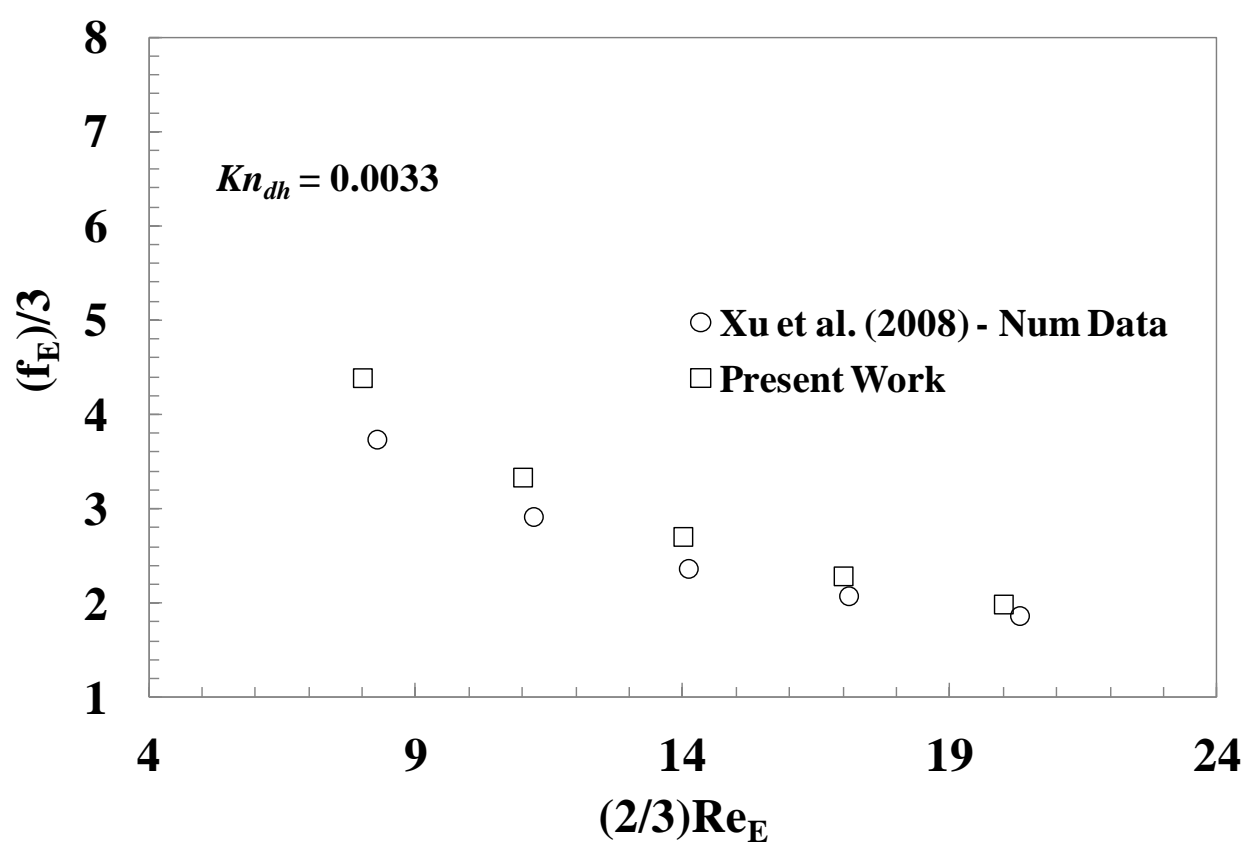

Figure 12: Friction factor comparison for rarefied flows with the data of Xu et al. (2008) 
In micro porous media, Knudsen numbers can vary over a wide range from continuum to slip and also transition regimes. Since the present study is focused on applications in the continuum and slip flow regime, friction factors are studied in detail by varying the Knudsen number from $0 \leq K n_{d h} \leq 0.1$. Fig. 13(a) plots the friction factors for SC beds at three different Knudsen number values. It can be seen that rarefaction has a huge influence on the friction factors. As the Knudsen numbers are increased, friction factors are significantly reduced at all Reynolds numbers. As explained earlier through velocity contour plots, velocity slip on the sphere surface increases and core velocities reduce due to rarefaction, which in turn results in smaller velocity gradients leading to lower pressure drop and/or friction factors. Similar behavior is observed for BCC and FCC beds as shown in Fig.13(b) and Fig.13(c) respectively, where friction factors are significantly reduced as rarefaction increases. It also appears that the reduction in friction factors is much higher for Knudsen variation from $K n_{d h}$ $=0$ to $K n_{d h}=0.05$, when compared to the reduction in the friction factors from $K n_{d h}=0.05$ to $K n_{d h}=0.1$

Since continuum correlations are inapplicable for rarefied flows, and also since there is a lack of generalized correlations for predicting pressure drop in both continuum and rarefied microporous media, we have proposed new improved generalized correlations obtained by curve-fitting our numerical data. Fig. 13 also lists the friction factors (dashed lines) calculated from our proposed correlations which are shown below in Eq. (17)-Eq. (19). Parameters for calculating rarefied flow friction factors are also listed in Table 2.

$$
\begin{aligned}
& \text { SC: } f_{E}=\frac{A_{E}}{\operatorname{Re}_{E}\left(1+C_{1} * \sqrt{K n_{d h}}\right)}+\frac{B_{E}}{\left(1+C_{2} * \sqrt{K n_{d h}}\right)}, \quad A_{E}=165, B_{E}=0.48, C_{1}=3, C_{2}=1.0 \\
& \text { BCC: } f_{E}=\frac{A_{E}}{\operatorname{Re}_{E}\left(1+C_{1} * \sqrt{K n_{d h}}\right)}+\frac{B_{E}}{\left(1+C_{2} * \sqrt{K n_{d h}}\right)}, \quad A_{E}=165, B_{E}=0.6, C_{1}=4, C_{2}=4.5 \\
& \text { FCC: } f_{E}=\frac{A_{E}}{\operatorname{Re}_{E}\left(1+C_{1} * \sqrt{K n_{d h}}\right)}+\frac{B_{E}}{\left(1+C_{2} * \sqrt{K n_{d h}}\right)}, \quad A_{E}=165, B_{E}=1.1, C_{1}=4.3, C_{2}=4.5
\end{aligned}
$$


Table 2

Parameters for evaluating modifying friction factor $\left(f_{E}\right)$ for infinite structured beds

\begin{tabular}{|c|c|c|c|c|}
\hline \multicolumn{5}{|c|}{ Continuum No-slip flows } \\
\hline Research Group & $A_{E}$ & \multicolumn{3}{|c|}{$\boldsymbol{B}_{E}$} \\
\hline $\begin{array}{c}\text { SC Beds: Yang et al. (2012) } \\
\text { Exp Data }\end{array}$ & 163.46 & \multicolumn{3}{|c|}{0.7425} \\
\hline $\begin{array}{c}\text { SC Beds: Yang et al. (2012) } \\
\text { Num Data }\end{array}$ & 161.865 & \multicolumn{3}{|c|}{0.66} \\
\hline $\begin{array}{c}\text { SC Beds: } \\
\text { Present Work - Eq.(14) }\end{array}$ & 165 & \multicolumn{3}{|c|}{0.48} \\
\hline $\begin{array}{c}\text { BCC Beds: Yang et al. } \\
\text { (2012) } \\
\text { Exp Data }\end{array}$ & 160 & \multicolumn{3}{|c|}{0.6075} \\
\hline $\begin{array}{c}\text { BCC Beds: Yang et al. } \\
\text { (2012) } \\
\text { Num Data }\end{array}$ & 146 & \multicolumn{3}{|c|}{0.2775} \\
\hline $\begin{array}{c}\text { BCC Beds: } \\
\text { Present Work - Eq. (15) }\end{array}$ & 165 & \multicolumn{3}{|c|}{0.6} \\
\hline $\begin{array}{c}\text { FCC Beds: Yang et al. } \\
\text { (2012) } \\
\text { Exp Data }\end{array}$ & 174 & \multicolumn{3}{|c|}{0.615} \\
\hline $\begin{array}{c}\text { FCC Beds: Yang et al. } \\
\text { (2012) } \\
\text { Num Data }\end{array}$ & 184.6 & \multicolumn{3}{|c|}{0.3375} \\
\hline $\begin{array}{c}\text { FCC Beds: } \\
\text { Present Work - Eq. (16) }\end{array}$ & 165 & \multicolumn{3}{|c|}{1.1} \\
\hline \multicolumn{5}{|c|}{ Rarefied Slip Flows } \\
\hline Research Group & $A_{E}$ & $\boldsymbol{B}_{E}$ & $C 1$ & $C 2$ \\
\hline $\begin{array}{c}\text { SC Beds: } \\
\text { Present Work - Eq. (17) }\end{array}$ & 165 & 0.48 & 3 & 1 \\
\hline $\begin{array}{c}\text { BCC Beds: } \\
\text { Present Work - Eq. (18) }\end{array}$ & 165 & 0.6 & 4 & 4.5 \\
\hline $\begin{array}{c}\text { FCC Beds: } \\
\text { Present Work }- \text { Eq. (19) }\end{array}$ & 165 & 1.1 & 4.3 & 4.5 \\
\hline $\begin{array}{l}\text { All Structured Beds: } \\
\text { Present Work - Eq. (20) }\end{array}$ & 165 & 0.48 & 3.8 & 1 \\
\hline
\end{tabular}




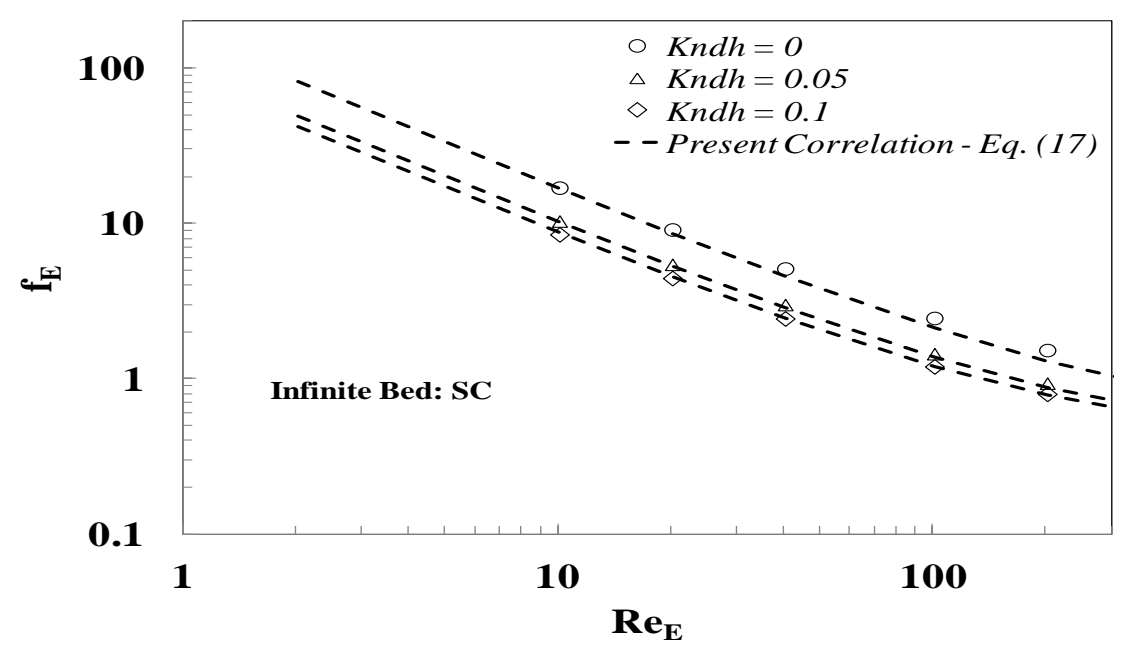

(a)

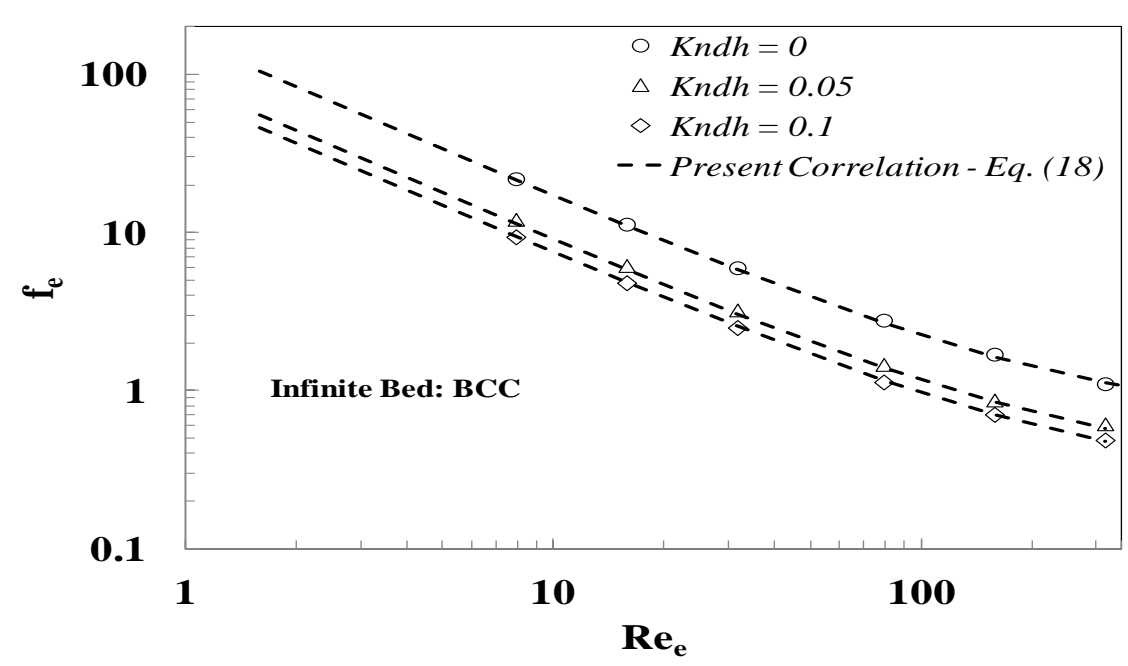

(b)

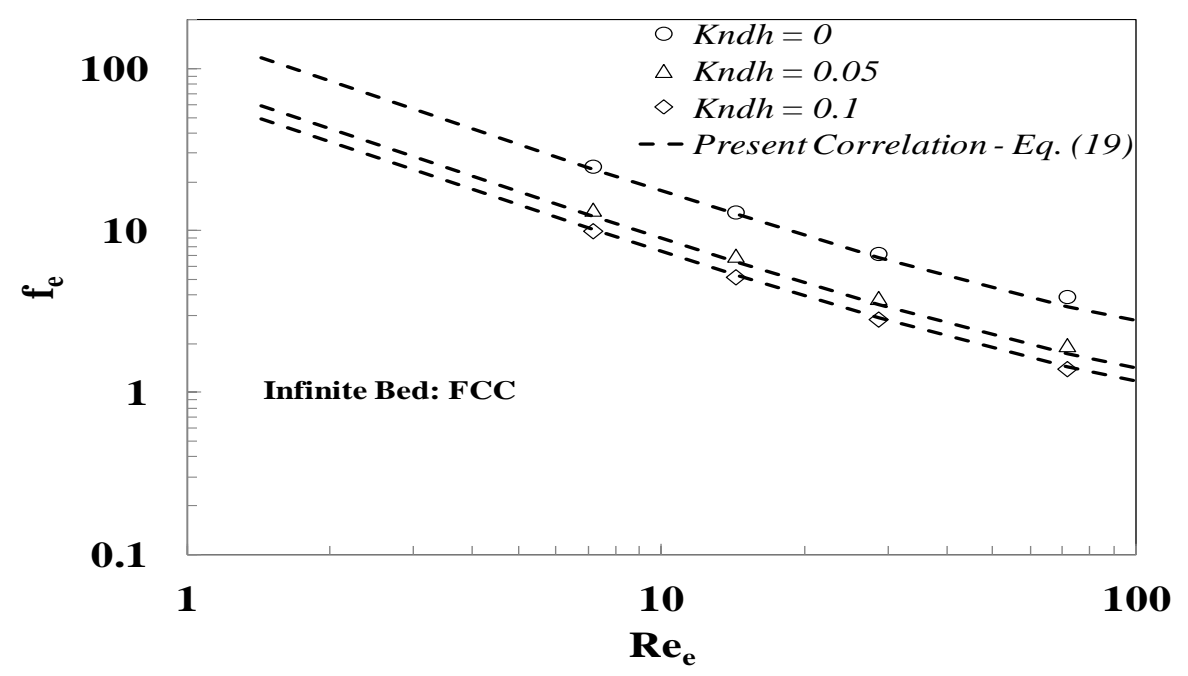

(c)

Figure 13: Rarefaction effect on friction factor for infinite structured beds (a) SC, (b) BCC, and (c) FCC 
Influence of packing form on the rarefied flow dynamics can be more clearly understood from Fig. 14, where friction factors for SC, BCC, and FCC beds are compared at different degrees of rarefaction. We have previously seen that for continuum macro-scale porous media flows, packing form has a significant influence at higher Reynolds numbers, particularly in the turbulent flow regime with FCC beds approaching the random beds. For micro porous media flows however, flow Reynolds numbers are very low and the flow is typically laminar. While results are presented in the present study up to $R e_{E}=100$, realistic Reynolds numbers for rarefied flows will be lower. As shown in Fig. 10(a), for $K n_{d h}=0$, friction factors for FCC beds are higher compared to BCC and SC beds for $\operatorname{Re}_{E}>30$. However, with increasing rarefaction as shown in Fig. 10(b) and Fig. 10(c), the packing form seems to have only a small influence with the friction factors for all three packing's following very closely with minor differences (5\% to $10 \%)$. Specifically, it appears that with increasing rarefaction, the tortuosity effect which resulted in higher pressure drop for FCC beds for continuum no-slip flows seems to become smaller and FCC beds behave more similarly to BCC and SC beds.

One significant observation from these studies that can be made is that for rarefied flows, packing arrangement or form is not very significant in terms of influencing resultant pressure drop. This observation is very important, because even though we have defined different correlations [Eq. (17)-Eq.(19)] for SC, BCC, or FCC beds based on curve-fitting our numerical data, a simplified single correlation may also be defined which can be used as a first approximation for predicting the friction factors for all three packing's (as shown below) for $R e_{E}<50$ for rarefied flows.

$$
f_{E}=\frac{A_{E}}{\operatorname{Re}_{E}\left(1+C_{1} * \sqrt{K n_{d h}}\right)}+\frac{B_{E}}{\left(1+C_{2} * \sqrt{K n_{d h}}\right)}, \quad A_{E}=165, B_{E}=0.48, C_{1}=3.8, C_{2}=1
$$




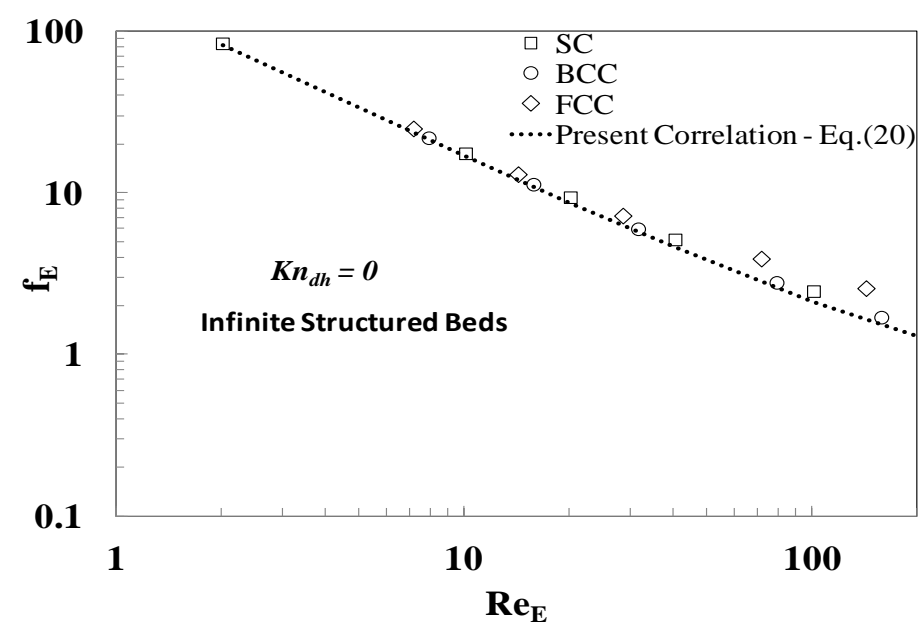

(a)

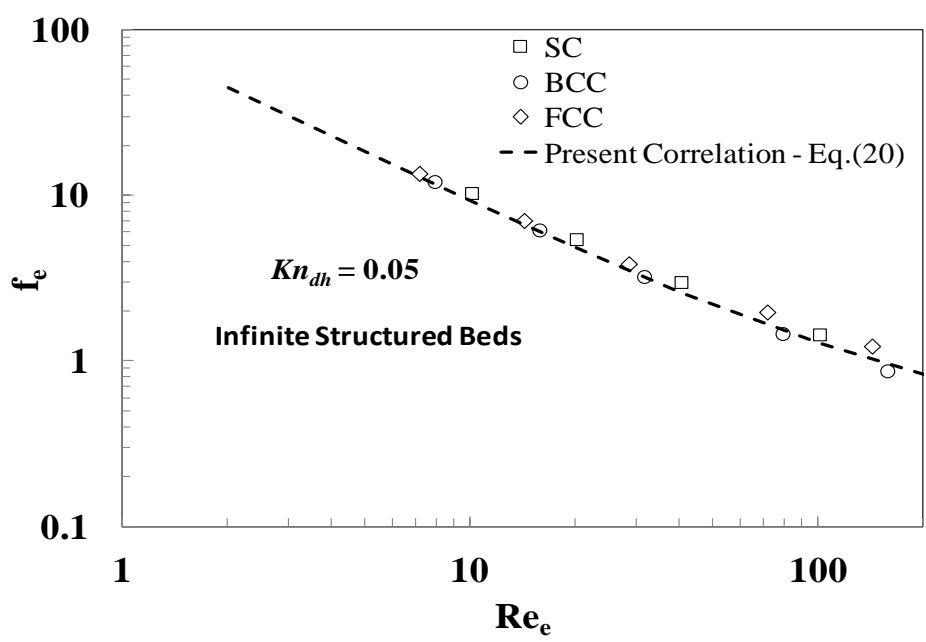

(b)

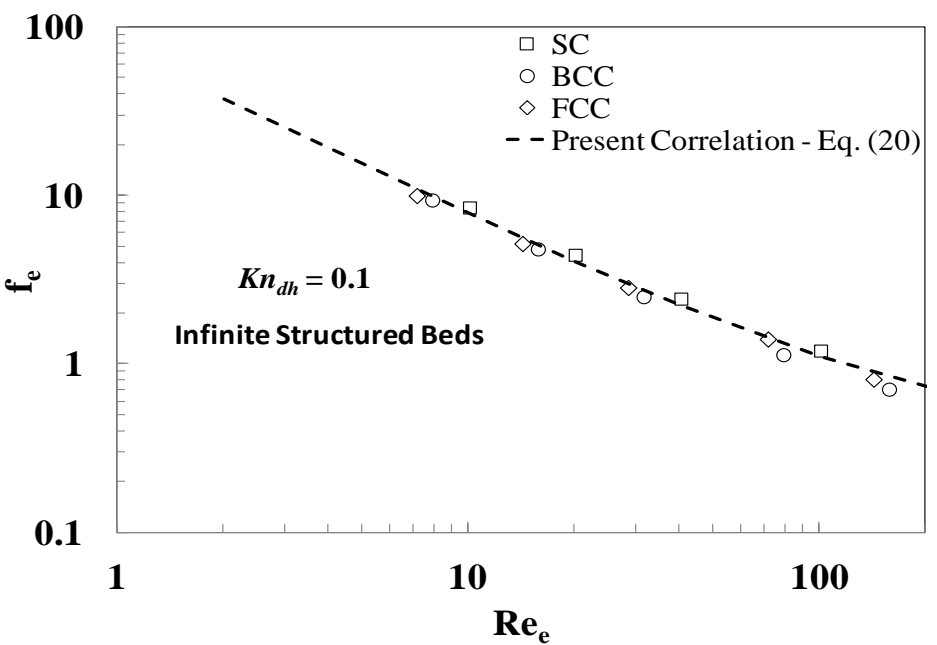

(c)

Figure 14: Packing arrangement influence on friction factors for rarefied flows at different Knudsen numbers. (a) $K n_{d h}=0$, (b), $K n_{d h}=0.05$ and (c) $K n_{d h}=0.1$ 
It may not be applicable for continuum no-slip flows as can be clearly seen from Fig. 14 (a), where the friction factors for FCC beds are still higher at higher Reynolds numbers and therefore detailed correlations should be used as in Eq. (17) to Eq. (19) which for $K n_{d h}=0$ will be reduced to Eq. (14) - Eq. (16) for continuum no-slip flows. For rarefied flows however, the simplified approximate correlation (with differences around 5 to $10 \%$ ) can be used for predicting the friction factors for the three packing forms.

\section{Conclusions}

No-slip continuum and also rarefied slip flows are successfully investigated in this work using hybrid FE/FV methods. Structured beds result in lower pressure drops compared to random beds due to the channeling effect present in the former. Therefore, Ergun correlation is not suitable for predicting friction factors for continuum flows in structured beds for $R e_{E}>$ 30. For continuum flows, it was found that, packing form has a significant influence in predicting the pressure drop, particularly for FCC beds. Due to the increase in tortuosity for BCC and FCC beds compared to SC beds, the fluid flow has to face much higher resistance, resulting in higher pressure drop for $\mathrm{BCC}$ and $\mathrm{FCC}$ beds in turbulent regime at higher Reynolds numbers. The tortuosity is highest for FCC beds, where friction factors are significantly higher, and trend towards the Ergun correlation predictions for random beds.

Rarefaction increases the slip velocity on sphere surfaces and lowers the core velocities in the channel, resulting in lower pressure drops compared to continuum flows. New generalized correlations are proposed in the study for infinite structured beds, which are applicable for continuum no-slip flows over a wide range of Reynolds numbers $\left(1<R e_{E}<\right.$ 4000), and also for rarefied micro porous media flows, which are applicable in the laminar regime $\left(\operatorname{Re}_{E}<300\right)$. Packing form has only a minor influence on rarefied flows with the differences in the results for friction factors due to the packing form around 5 to $10 \%$ in the entire laminar flow regime. 


\section{Acknowledgement}

This work is funded by Engineering Research and Development Center (ERDC) through the Department of Defense research programs.

\section{Nomenclature}

$a_{w} \quad$ Wetted area per unit volume of packed bed

A cross-sectional area available for flow

$A_{T} \quad$ total cross-sectional area of the bed

$A_{E} \quad$ Parameter for calculating friction factor

$B_{E} \quad$ Parameter for calculating friction factor

$c_{b 1,} c_{b 2} c_{w 1} \quad$ Model parameters in Spalart-Allmaras equation

$C_{s p} \quad$ Contact spacing between spheres

$d_{p} \quad$ sphere particle diameter

$d_{h} \quad$ Hydraulic diameter of the porous media

$f_{E} \quad$ Pore-scale friction factor based on hydraulic diameter

$f n_{w} \quad$ Model parameter in Spalart-Allmaras equation

Kn $\quad$ Knudsen number

$K n_{d p} \quad$ Knudsen number based on particle diameter

$K n_{d h} \quad$ Knudsen number based on hydraulic diameter

$L \quad$ Length of the bed

pressure

$\Delta P \quad$ pressure drop

$Q \quad$ volumetric flow rate

$q \quad$ auxiliary variable closely related to pressure

$q^{\prime} \quad$ incremental value of $\mathrm{q}$ 


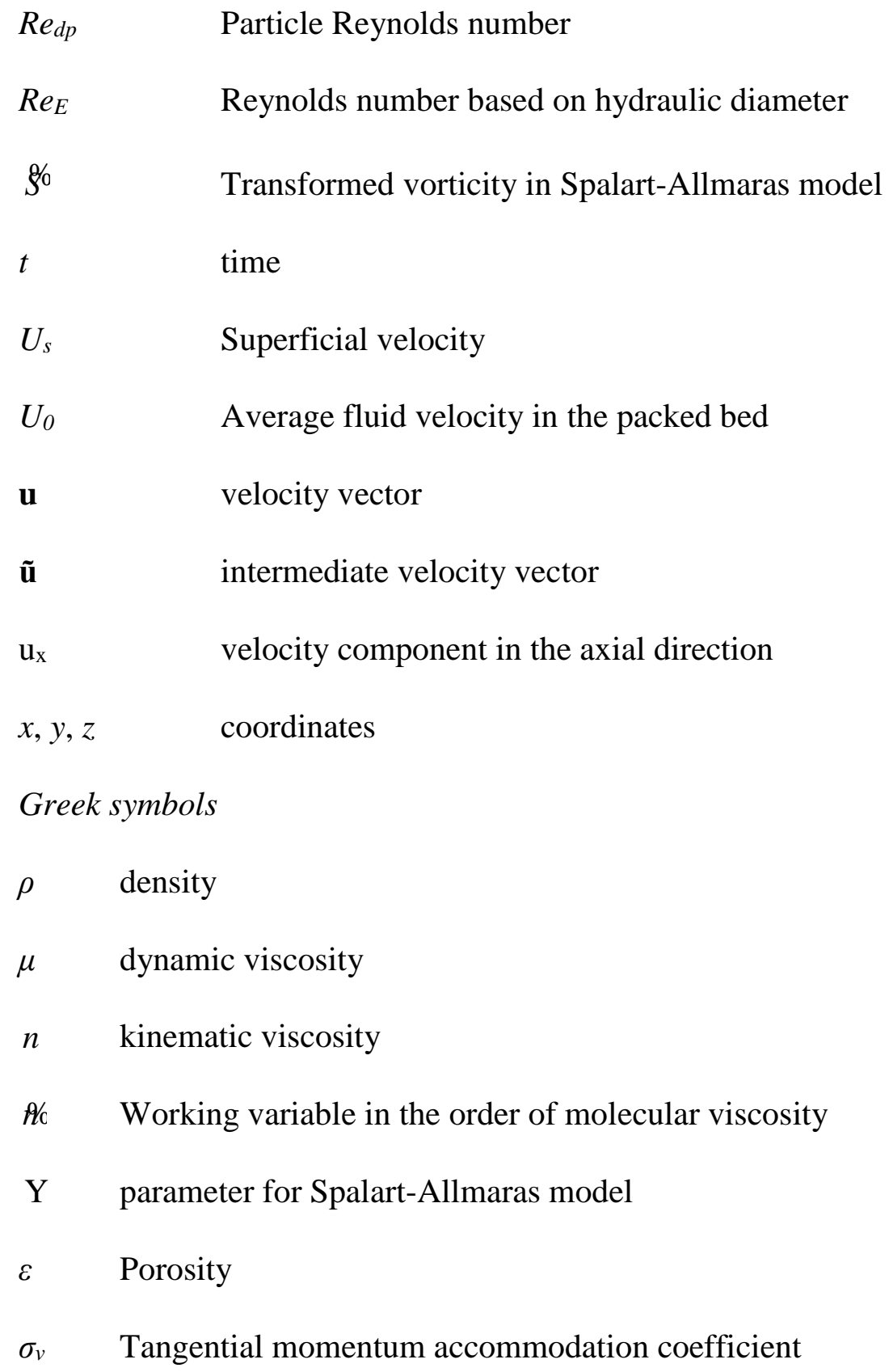

\section{REFERENCES}

[1] Calis HPA, Nijenhuis J, Paikert BC, Dautzenberg FM, van den Bleek CM. CFD modelling and experimental validation of pressure drop and flow profile in a novel structured catalytic reactor packing. Chemical Engineering Science 2001; 56 (4): 17131720. 
[2] Cybulski, A, Moulijn JA. Structured catalysts and reactors. Newyork: Marcel Dekker Inc; 1998.

[3] Yang J, Wang Q, Zeng M, Nakayama A. Computational study of forced convective heat transfer in structured packed beds with spherical or ellipsoidal particles. Chemical Engineering Science 2010; 65: 726-738

[4] Yang J, Wang J, Bu S, Zeng M, Wang Q, Nakayama A. Experimental analysis of forced convective heat transfer in novel structured packed beds of particles. Chemical Engineering Science 2012; 71: 126-137

[5] Xu RN, Jiang PX. Numerical simulation of fluid flow in microporous media. International Journal of Heat and Fluid Flow 2008; 29: 1447-1455.

[6] Tang GH, Tao WQ, He YL. Three dimensional lattice Boltzmann model for gaseous flow in microducts and microscale porous media. Journal of Applied Physics 2005; 97: 104918.

[7] Ergun S. Fluid flow through packed columns. Chemical Engineering Progress 1952; 48: 89-94.

[8] Nijemeisland M, Dixon AG, Comparison of CFD simulations to experiment for convective heat transfer in a gas-solid fixed bed. Chemical Engineering Journal 2001; 82: $231-246$.

[9] Nijemeisland M, Dixon AG. CFD Study of fluid flow and wall heat transfer in a fixed bed of spheres. AIChE Journal 2004; 50: 906-921.

[10] Eisfeld B, Schnitzlein K. The influence of confining walls on the pressure drop in packed beds. Chemical Engineering Science 2001; 56: 4321-4329.

[11] Reichelt W. Zur Berechnung des Druckverlustes einphasig durchstroK mter Kugel- und ZylinderschuK ttungen. Chemie-Ingenieur-Technik, 1972; 44: 1068-1071. 
[12] Magnico P. Hydrodynamic and transport properties of packed beds in small tube-tosphere diameter ratio: pore scale simulation using an Eulerian and a Lagrangian approach. Chemical Engineering Science 2003; 58, 5005-5024.

[13] Guardo A, Coussirat M, Larrayoz M. Influence of the turbulence model in CFD modeling of wall-to-fluid heat transfer in packed beds. Chemical Engineering Science 2005; 60: $1733-1742$.

[14] Guardo A, Coussirat M, Larrayoz MA, Recasens F, Egusquiza E. Influence of the turbulence model in CFD modeling of wall-to-fluid heat transfer in packed beds. Chemical Engineering Science 2005; 60(6): 1733-1742.

[15] Atmakidis T, Kenig EY. CFD-based analysis of the wall effect on the pressure drop in packed beds with moderate tube/particle diameter ratios in the laminar flow regime. Chemical Engineering Journal 2009; 155: 404-410.

[16] Zhavoronkov NM, Aerov ME, Umnik NN. Hydraulic resistance and density of packing of a granular bed. J. Phys. Chem. 1949; 23: 342-361.

[17] Augier F, Idoux F, Delenne JY. Numerical simulations of transfer and transport properties inside packed beds of spherical particles. Chemical Engineering Science 2010; 65: $1055-1064$.

[18] Baker MJ, Tabor GR. Computational analysis of transitional air flow through packed columns of spheres using finite volume technique. Computers and Chemical engineering 2010; 34: 878-885.

[19] Cheng NS. Wall effect on pressure drop in packed beds. Powder Technology 2011; 210: 261-266.

[20] Susskind H, Becker W. Pressure drop in geometrically ordered packed beds of spheres. AIChE J. 1967; 13(6): 1155-1159. 
[21] Romkes SJP, Dautzenberg FM, van den Bleek CM, Calis HPA. CFD modelling and experimental validation of particle-to-fluid mass and heat transfer in a packed bed at very low channel to particle diameter ratio. Chem. Eng. J. 2003; 96(1-3): 3-13.

[22] Gunjal PR, Ranade VV, Chaudhari RV. Computational study of a single- phase flow in packed beds of spheres. AIChE J. 2005; 51(2): 365-378

[23] Choi HS, Park HC, Huh C, Kang SG. Numerical simulation of fluid flow and heat transfer of supercritical $\mathrm{CO}_{2}$ in micro-porous media. Energy Procedia 2011; 4: 3786-3793

[24] Li H, Qiu S, Zhang Y, Su G, Tian W. Thermal hydraulic investigations with different fuel diameters of pebble bed water cooled reactor in CFD simulation. Annals of Nuclear Energy 2012; 42: 135-147

[25] Palle S, Aliabadi S. Direct simulation of structured wall bounded packed beds using hybrid FE/FV methods. Computers and Fluids, Accepted May 29, 2013, http://dx.doi.org/10.1016/j.compfluid.2013.05.012.

[26] Civan F. A review of approaches for describing gas transfer through extremely tight porous media. Porous Media and Its Applications in Science, Engineering, and Industry $3^{\text {rd }}$ International conference, 2010. American Institute of Physics.

[27] Jeong N, Choi DH, Lin CH. Prediction of Darcy-Forchheimer drag for micro-porous structures of complex geometry using the lattice Boltzmann method. J. Micromech. Microeng 2006; 16: 2240-2250.

[28] Maxwell JC. On stresses in rarefied gases arising from inequalities of temperature. Philosophical Transactions of the Royal Society 1879; Part1, 170: 231-256.

[29] Yu S, Ameel TA. Slip-flow heat transfer in rectangular microchannels. Int. J. Heat Mass Transfer 2001; 44; 4225-4234. 
[30] Spalart PR, Jou WH, Strelets M, and Allmaras SR. Comments on the feasibility of LES for wings and on hybrid RANS/LES approach. Proceedings of the $1^{\text {st }}$ AFOSR International Conference on DNS/LES 1997; Columbus, OH.

[31] Aliabadi S, Tu S. Hybrid finite element / finite volume flow solver for flow and transport problems. 14th International Conference on Finite Elements in Flow Problems 2007; Santa Fe, New Mexico, March 26-28.

[32] Tu S, Aliabadi S. A Hybrid finite volume/element method for low and high Reynolds number incompressible Flows. Abstract of 8th World Congress on Computational Mechanics (WCCM8) and 5th. European Congress on Computational Methods in Applied Sciences and Engineering (ECCOMAS) 2008; June 30 - July 5, Venice, Italy.

[33] Tu S, Aliabadi, S. Development of a hybrid finite volume/element solver for incompressible flows on unstructured meshes. International Journal of Numerical methods in Fluids 2007; 55(2): 177-203.

[34] Barber RW, Emerson DR. Challenges in Modeling Gas-Phase Flow in Microchannels: From Slip to Transition. Heat Transfer Engineering 2006; 27 (4): 3-12. 